\title{
Aging impairs the essential contributions of non-glial progenitors to neurorepair in the dorsal telencephalon of the Killifish $N$. furzeri
}

\author{
1 Jolien Van houcke ${ }^{1}$, Valerie Mariën ${ }^{1}$, Caroline Zandecki ${ }^{2}$, Sophie Vanhunsel ${ }^{3}$, Lieve \\ 2 Moons ${ }^{3,4}$, Rajagopal Ayana ${ }^{1,2}$, Eve Seuntjens ${ }^{2,4}$, Lutgarde Arckens ${ }^{1,4}{ }^{*}$ \\ $3{ }^{1}$ Laboratory of Neuroplasticity and Neuroproteomics, Department of Biology, KU Leuven, 3000 \\ 4 Leuven, Belgium \\ 5 '2Laboratory of Developmental Neurobiology, Department of Biology, KU Leuven, 3000 Leuven, \\ 6 Belgium \\ $7 \quad{ }^{3}$ Laboratory of Neural Circuit Development and Regeneration, Department of Biology, KU \\ 8 Leuven, 3000 Leuven, Belgium \\ $9{ }^{4}$ Leuven Brain Institute, 3000 Leuven, Belgium \\ $10{ }^{*}$ Correspondence: lut.arckens@kuleuven.be
}

11 Highlights

12 - Aging impairs neurorepair in the killifish pallium at multiple stages of the regeneration 13 process

- Atypical non-glial progenitors support the production of new neurons in the naive and injured dorsal pallium

- The impaired regeneration capacity of aged killifish is characterized by a reduced reactive proliferation of these progenitors followed by a decreased generation of newborn neurons that in addition, fail to reach the injury site

- Excessive inflammation and glial scarring surface as potential brakes on brain repair in the aged killifish pallium

\section{Summary}

23 The aging central nervous system (CNS) of mammals displays progressive limited regenerative abilities. Recovery after loss of neurons is extremely restricted in the aged brain. Many research models fall short in recapitulating mammalian aging hallmarks or have an impractically long lifespan. We established a traumatic brain injury model in the African turquoise killifish (Nothobranchius furzeri), a regeneration-competent vertebrate model that evolved to naturally age extremely fast. Stab-wound injury of the aged killifish dorsal telencephalon unveils an impaired and incomplete regeneration response when compared to young individuals. Remarkably, killifish brain regeneration is mainly supported by atypical non-glial progenitors, yet their proliferation capacity appears declined with age. We identified a high inflammatory 
response and glial scarring to also underlie the hampered generation of new neurons in aged fish. These primary results will pave the way for further research to unravel the factor age in relation to neurorepair, and to improve therapeutic strategies to restore the injured and/or diseased aged mammalian CNS.

\section{Keywords}

Aging, neuroregeneration, neurorepair, non-glial progenitors (NGPs), Nothobranchius furzeri, Killifish, traumatic brain injury, neurodegenerative diseases

\section{Introduction}

Age-related neurodegenerative diseases are highly debilitating and incurable pathologies that impinge a high socio-economic burden on our society (El-Hayek et al., 2019). They share a progressive degeneration of neurons, which results in loss of brain function and a heterogeneous array of incapacitating symptoms (Dugger et al., 2017). Therapeutic strategies for brain restoration consist of compensating for neuronal loss by generating new neurons from the existing stem cell pools that can integrate in the existing circuitry. The capacity for neuroregeneration is naturally limited in the adult mammalian brain (Tanaka et al., 2009; Zhao et al., 2016). Neural stem cells may start dividing upon injury, but a large part of the newly generated neurons fail to mature, survive and integrate into the existing neural network, thereby restricting full recovery (Arvidsson et al., 2002; Thored et al., 2006; Kernie et al., 2010; Turnley et al., 2014; Grade et al., 2017). The mammalian neurogenic potential diminishes even further with advancing age, which constitutes one of the main risk factors for neurodegenerative diseases (Galvan et al., 2007; Popa-Wagner et al., 2011; Hou et al., 2019). Studying vertebrate aging in models with high neuroregenerative capacities, such as teleost fish, can therefore help reveal key information to deal with each of these physiological brakes on neuroregeneration and neurorepair (Zhao et al., 2016).

Teleost fish share approximately $70 \%$ of the coding genes with mammals (Howe et al., 2013), but in contrast to mammals, retain the ability to regenerate multiple organs, including fin, heart and the central nervous system (CNS) (Zupanc, 2001; Zupanc et al., 2012; Wendler et al., 2015; Marques et al., 2019; Zambusi et al., 2020; Van houcke et al., 2021). They have been extensively exploited as gerontology models in the past (Ding et al., 2010; Gopalakrishnan et al., 2013; Van houcke et al., 2015, 2021; Kim et al., 2016; Platzer et al., 2016). Yet, most teleosts are relatively long-lived (3-5 years) just like mice (Gerhard et al., 2002; Miller et al., 2002; Gopalakrishnan et al., 2013), making investigations about the specific impact of age impractical (Van houcke et al., 2021). The African turquoise killifish (N. furzeri) however, has surfaced as an ideal vertebrate model for aging studies because of its extremely short lifespan. The short-lived GRZ stain for example, has a median lifespan of 4-6 months depending on housing conditions (Valdesalici et al., 2003; Reichwald et al., 2015; Valenzano et al., 2015; Polačik et al., 2016). Killifish naturally 
live in ephemeral ponds in Africa, which have forced this species to evolve into a short lifespan and rapid aging (Genade et al., 2005). Many typical aging hallmarks of mammals are conserved in killifish (Kim et al., 2016; Platzer et al., 2016; Hu et al., 2018; Van houcke et al., 2021). Understanding how aged killifish retain or lose their high regenerative capacity upon CNS aging, could thus catalyze the development of therapeutic strategies aiming at inducing successful neuroregeneration in the adult mammalian brain.

74 The telencephalon or forebrain of fish is a favorable model of study since homologues to the two main neurogenic zones of mammals, the subgranular zone (SGZ) of the hippocampus and the subventricular zone (SVZ) of the lateral ventricles, have been identified in the pallium and subpallium (Adolf et al., 2006; Mueller et al., 2009, 2011). It was recently discovered that the killifish dorsal pallium holds two classes of progenitors: (1) the commonly known radial glia (RGs) and (2) the non-glial progenitors (NGPs). NGPs are devoid of the typical astroglial/RG markers, such as glutamine synthetase (GS), brain lipid-binding protein (BLBP), glial fibrillary acidic protein (GFAP) and vimentin, and have a morphology that is less branched than RGs (Coolen et al., 2020). Early in development, killifish RGs enter a premature quiescent state and proliferation is supported by the NGPs. This is in sharp contrast to the situation in zebrafish, where RGs represent the neurogenic population in the dorsal pallium (Kroehne et al., 2011; Rothenaigner et al., 2011; Coolen et al., 2020). How these two different progenitor classes, with the NGPs appearing unique to the killifish, behave in the neuroregenesis process, and whether their neurogenic capacity is influenced by aging, remains unexplored.

In the present study, we have first set up and validated stab-wound injury as a reliable traumatic brain injury (TBI) model. Next, we have decoded the impact of aging on the regeneration capacity - and on the two neurogenic pools - in the killifish dorsal pallium after stab-wound injury. We find that aged killifish, just like mammals, are not capable to successfully regenerate; they develop an excessive inflammatory reaction and glial scarring, show diminished injury-induced neurogenesis, and the vast majority of newborn neurons fail to reach the injury site. Remarkably, we show neuroregeneration to be supported by the NGPs in both young adult and aged killifish, and not the RGs that typically constitute neuroregenesis in other teleosts. Taken together, aged killifish appear to mimic the impaired regeneration capacity also seen in adult and/or aged mammals, instead of displaying the high regenerative capacities seen in young adult teleost fish, including killfish. In summary, we propose the killifish aging nervous system as a valuable model to create knowledge about the identity and mode of action of drivers and brakes of neuroregenerative properties. This may eventually elucidate how to boost the repair capacity in an aging context in the diseased/injured mammalian brain. 


\section{Methods}

\section{RESOURCE AVAILABILITY}

\section{Lead contact and Materials availability}

106 Further information and requests for resources (killifish) should be directed to and will be fulfilled

107 by the Lead Contact, Lutgarde Arckens (lut.arckens@kuleuven.be).

\section{Data and code availability}

109 The present study has no unique datasets or code.

\section{EXPERIMENTAL MODEL AND SUBJECT DETAILS}

\section{Fish strain and housing}

112 All experiments were performed on adult (6 week- and 18 week-old) female African turquoise 113 killifish (Nothobranchius furzeri), inbred strain GRZ-AD, which were kindly provided by Prof. Dr.

114 L. Brendonck and Dr. T. Pinceel and originate from the Biology of Ageing, Leibniz Institute for Age 115 Research - Fritz Lipmann Institute, Jena, Germany. Breeding pairs were housed in $8 \mathrm{~L}$ aquaria and 116 experimental fish were kept in 3,5 L aquaria in a ZebTEC Multi-Linking Housing System 117 (Tecniplast). One male was housed with three females under standardized conditions; 118 temperature $28^{\circ} \mathrm{C}, \mathrm{pH} \mathrm{7}$, conductivity $600 \mu \mathrm{s}, 12 \mathrm{~h} / 12 \mathrm{~h}$ light/dark cycle, and fed twice a day with 119 (Artemia salina, Ocean Nutrition) and mosquito larvae (Chironomidae, Ocean Nutrition). 120 Breeding pairs were given sandboxes for spawning. Fertilized eggs were collected once a week 121 and washed with methylene blue solution (0,0001\% in autoclaved system water, Sigma-Aldrich, 122 03978) for five minutes. Next, eggs were bleached twice for five minutes in 1\% Hydrogen 123 Peroxide $\left(\mathrm{H}_{2} \mathrm{O}_{2}\right.$, Chem-Lab, CL00.2308.5000) diluted in autoclaved system water. Then, eggs were 124 again washed four times for five minutes with methylene blue solution. Eggs were stored on 125 moist Jiffy-7C coco substrate plates (Jiffy Products International AS, Norway) at $28^{\circ} \mathrm{C}$ with a $12612 \mathrm{~h} / 12 \mathrm{~h}$ light/dark cycle for three weeks in a custom-made incubator. Embryos that reached the 127 'Golden Eye stage' (Polačik et al., 2016) were hatched in a small volume of ice-cold Humic acid 128 solution (Sigma-Aldrich, 53680, 1g/L in system water) with continuous oxygenation. Larvae were 129 raised at $26^{\circ} \mathrm{C}$ and half of the water was changed daily for one week. Hereafter, larvae were 130 transferred to 3,5 L aquaria and fed daily with (Artemia salina, Ocean Nutrition) until 3 weeks 131 post hatching. All experiments were approved by the KU Leuven ethical committee in accordance 132 with the European Communities Council Directive of 22 September 2010 (2010/63/EU) and the 133 Belgian legislation (KB of 29 May 2013).

\section{Method details}

\section{Stab-wound injury}


136 Fish were first sedated in 0,03\% buffered tricaine (MS-222, Sigma-Aldrich, CAS: 886-86-2), diluted

137 in system water, and placed in a cold moist sponge. Scales, skin and fat tissue was removed above 138 the right telencephalic hemisphere to visualize the skull. Next, a custom Hamilton 33-Gauge 139 needle was pushed through the skull into the dorsal pallium of the right hemisphere of the 140 telencephalon, causing a brain lesion of approximately a depth of $500 \mu \mathrm{m}$ (Figure S1). The 141 telencephalon lies in between the eyes of the killifish, which were used as landmarks. The needle 142 was dipped in Vybrant DiD cell-labeling solution $\left(\mathrm{C}_{67} \mathrm{H}_{103} \mathrm{ClN}_{2} \mathrm{O}_{3} \mathrm{~S}\right.$, Thermo Fisher Scientific, $143 \mathrm{~V} 22887$ ) to easily find the place of entrance and needle track on sections (Figure S1). Afterwards, 144 the fish were placed in fresh system water to recover.

\section{BrdU labeling}

146 To label dividing cells and their progeny, fish were placed in 5-Bromo-2'-deoxyuridine (BrdU, 147 Sigma-Aldrich, B5002-5G; CAS: 59-14-3) water (7,5 mM in system water) for 16 hours between 148 one and two days post injury. After the pulse, fish were placed in fresh system water for a chase 149 period of 21 days.

\section{Tissue fixation and processing}

151 Fish were euthanized in 0.1\% buffered tricaine (MS-222, Sigma-Aldrich, diluted in system water) 152 and perfused via the heart with PBS and 4\% paraformaldehyde (PFA, Sigma-Aldrich, 8.18715, CAS 153 30525-89-4, diluted in PBS). Brains were extracted and fixed for 12 hours in $4 \%$ PFA at $4{ }^{\circ} \mathrm{C}$. 154 Afterwards, brains were washed three times with PBS and embedded in 30\% sucrose, 1,25\% 155 agarose in PBS. Coronal sections of $10 \mu \mathrm{m}$ were made on a CM3050s cryostat (Leica) and collected 156 on SuperFrost Plus Adhesion slides (Thermo Fisher Scientific, 10149870). Sections were stored at $157-20^{\circ} \mathrm{C}$ until immunohistochemistry (IHC) or Cresyl Violet staining.

\section{Cresyl Violet histological staining}

159 Cryostat sections were dried for 30 minutes at $37^{\circ} \mathrm{C}$ to improve adhesion to the glass slides and 160 washed in aqua destillata (AD). Next, the sections were immersed in Cresyl Violet solution (1\% in 161 AD, Fluka Chemicals, Sigma-Aldrich) for five minutes. The sections were rinsed in $200 \mathrm{~mL}$ AD with 162 five drops of Acetic Acid (Glacial, 100\%) for 30 seconds. Hereafter, the sections were dehydrated 163 in $100 \%$ ethanol and $100 \%$ xylol series. Sections were covered with DePeX and cover slip and 164 dried overnight.

\section{Immunohistochemistry (IHC)}

166 Sections were dried for half an hour at $37^{\circ} \mathrm{C}$ to improve adhesion to the glass slides and washed 167 in AD and TBS (0,1\% Triton-X-100 in PBS). Heat-mediated antigen retrieval was used to break 168 cross-links between the proteins. The slices were boiled in the microwave in $1 \mathrm{X}$ citrate buffer $(2,1$ 169 g citric acid and $500 \mu \mathrm{L}$ Tween 20 in $1 \mathrm{~L}$ PBS, pH 6) for five minutes at $100 \%$ and two times five 170 minutes at $80 \%$. Afterwards, the slices were cooled down for 20 minutes and washed three times 
171 for five minutes with TBS. For $\mathrm{BrdU} \mathrm{IHC}$, sections were pretreated with $2 \mathrm{~N} \mathrm{HCl}$ at $37^{\circ} \mathrm{C}$ for 30

172 minutes to break the DNA and washed with $0,1 \mathrm{M}$ sodium borate (in $\mathrm{AD}$ ) to neutralize $\mathrm{HCl}$.

173 Sections were blocked for one hour at room temperature with 20\% normal goat serum (Sigma-

174 Aldrich, S26) in Tris- NaCl blocking buffer (TNB). For IHC stainings involving the primary antibody

175 Goat anti-BLBP (Abcam, ab110099), blocking was performed with normal donkey serum (Sigma-

176 Aldrich, S30). Sections were stained over night with primary antibodies diluted in TNB at room

177 temperature, with the exception of the anti-HuC/D antibody, which was incubated at $4^{\circ} \mathrm{C}$ for 48

178 hours in Pierce Immunostain Enhancer (Thermo Fisher Scientific, 46644). Pierce Enhancer was

179 also used in triple IHC stainings and when the anti-L-plastin primary antibody (GeneTex,

180 GTX124420) was involved. The primary antibodies used were rabbit anti-SOX2 (1:1000, Sigma-

181 Aldrich, SAB2701800), mouse anti-HuC/D (1/200, Thermo Fisher Scientific, A-21271), mouse anti-

182 PCNA (1:500, Abcam, ab29), Goat anti-BLBP (1:1000, Abcam, ab110099), rat anti-BrdU (1:1000,

183 Abcam, ab6326), rabbit anti-L-plastin (1:400, GeneTex, GTX124420) and mouse anti-GS (1:1000,

184 Abcam, ab64613). Sections were washed with TBS three times for five minutes. Secondary

185 antibodies were stained at room temperature in TNB for two hours (Alexa-594, Alexa-488, Alexa-

186 305, 1:300, Thermo Fisher Scientific). In case of the anti-L-plastin IHC staining, a long

187 amplification was used, in which the secondary antibody is coupled to biotin (Goat anti-Rabbit-

188 biotin, 1:300 in TNB, Agilent Dako, E043201-8) and incubated for 45 minutes. After washing three

189 times for five minutes with TBS, Streptavidin-Cy5 (1/500 in TNB, Thermo Fisher Scientific,

190 SA1011) was added to the sections for two hours. For cell death detection, the TUNEL assay (In

191 Situ Cell Death Detection Kit, Fluorescein, Sigma-Aldrich, 11684795910) was used, following the

192 manufacturer's instructions. Cell nuclei were stained with 4',6-diamidino-2-fenylindool (DAPI, 193 1:1000 in PBS, Thermo Fisher Scientific). Last, sections were covered with Mowiol solution and a 194 cover glass slide.

\section{Microscopy}

196 Sections were scanned for DiD positivity using Texas Red light with a confocal microscope 197 (FV1000, Olympus), to locate the injury site, even after neuroregeneration was completed. For 198 the quantification of IHC stainings and Cresyl Violet staining, a Zeiss ('Axio Imager Z1') 199 fluorescence microscope was used to photograph three sections per animal. For fluorescence 200 pictures the AxioCam MR R3 camera was used. For Bright field pictures, the Mrc5 color camera 201 was used. 20X Tile scans were taken and stitching was performed using the ZEN software (ZEN 202 Pro 2012, Carl Zeiss). For greater detail, 63X magnification pictures were taken using immersion 203 oil. Channels were equally intensified for all conditions using Adobe Photoshop SC5 (Adobe 204 Systems) for publication. Figure configurations were made with Adobe Illustrator (Adobe 205 Systems).

206 QUANTIFICATION AND STATISTICAL ANALYSIS 
207 Immunopositive cells were counted (Figure S4) and the injury area surface $\left(\mathrm{mm}^{2}\right)$ was measured 208 (Figure S2) on three sections adjacent to the lesion site and compared with corresponding 209 sections of naive animals using respectively the cell counter plugin and polygon tool in Image J 210 (Fiji). An average of three sections was taken per animal for statistical analysis using GraphPad 211 Prism (version 8.2.1).

212 Data were analyzed by two independent observers and first tested for Gaussian normality and 213 homoscedasticity. If these assumptions were met, we used a parametric unpaired $t$-test (two 214 conditions) or one-way ANOVA (> two conditions), followed by Dunnett's multiple comparisons 215 test to compare injured fish to naive fish for each age separately. If these assumptions were not 216 met, we used the non-parametric Mann Whitney test (two conditions) or Kruskal-Wallis test (> 217 two conditions), followed by Dunn's multiple comparisons test. Two-way ANOVA was used to 218 compare young and aged fish at each time point, followed by Sidak's multiple comparisons test. $219 \mathrm{n}$ represents the number of animals in each condition. All values are mean \pm standard error of 220 the mean (SEM). Means were statistically significantly different when $\mathrm{p} \leq 0,05$.

221 Results

222 A stab-wound injury model to study neuroregeneration in the killifish pallium

223 Before studying neuroregeneration in the young and aged dorsal pallium of killifish, we first introduced an easy-to-use and reproducible brain injury model. Stab-wound injuries have been extensively characterized in the zebrafish telencephalon and effectively induce brain regeneration (Ayari et al., 2010; Kroehne et al., 2011; März et al., 2011; Baumgart et al., 2012; Kishimoto et al., 2012; Kyritsis et al., 2012; Barbosa et al., 2015). We chose to optimize a similar injury model in the young adult ( 6 week-old) and aged (18 week-old) killifish telencephalon (Figure S1A-B). Approaching the dorsal telencephalon through the nostrils was not possible without damaging the large eyes, since the nostrils of the killifish are positioned more lateral compared to zebrafish. Instead, the medial part of the right telencephalic hemisphere was targeted from above, and a 33-gauge Hamilton needle was pushed through the skull, approximately $500 \mu \mathrm{m}$ in depth (Figure S1C). The medial telencephalon lies in between the eyes, which were used as visual landmarks. Skin and fat tissue cover the skull of the killifish and were removed to visualize the skull of the fish. The procedure required an experienced hand and a training period. Just prior to wounding, the needle was dipped in Vybrant DiD Cell-Labeling Solution (Thermo Fischer) in order to permanently label the cells close to the injury site. This DiD dye approach enabled reconstruction of the original injury site, even if complete recovery had taken place, and the wounded area could no longer be distinguished from the surrounding tissue by (immuno)histology (Figure S1D,E). 


\section{Aging impairs tissue recovery after stab-wound injury}

243 Standard histology revealed a packed blood clot that filled the injury site in the brain of young

244 adult and aged killifish immediately upon injury (Figure 1A,B). In ensuing weeks, the parenchyma 245 of the young adult telencephalon was able to structurally regenerate in a seamless fashion, 246 showing a normal distribution of cells even at the DiD-positive injury site, 23 to 30 days post

247 injury (dpi) (Figure 1C, see also Figure S1F). In aged killifish on the contrary, the parenchyma 248 showed a malformation, recognizable by swollen and irregularly-shaped cells and blood vessels 249 at $30 \mathrm{dpi}$. The malformation was reminiscent of a mammalian glial scar (Figure 1D, see also Figure 250 S1G). We tested if the scar tissue, only observed in aged killifish, was of glial nature. As the teleost 251 telencephalon is devoid of parenchymal astrocytes (Grupp et al., 2010), we probed for the 252 presence of microglia and the long bushy fiber of RGs $\left(\mathrm{GS}^{+}\right)$that span the entire parenchyma. At $25323 \mathrm{dpi}$ a cluster of L-plastin ${ }^{+}$microglia/macrophages was present at the injury site, co-localising 254 with the scar tissue and the DiD labeled cells in aged killifish. Even at $30 \mathrm{dpi}$, the 255 microglia/macrophage cluster was still present in aged injured killifish (Figure S1F-I) indicating 256 that the scar is likely permanent. We did not observe RG cell bodies at the scar tissue. However, 257 the $\mathrm{GS}^{+}$fibers of the RG were surrounding the glial scar, indicating that RGs were still involved in 258 glial scarring via the use of their bushy fiber.

259 In conclusion, stab-wound injury could effectively be applied to compare the regeneration 260 process between young and aged killifish brains. Aged killifish brain recovered incompletely and 261 showed signs of permanent glial scarring.

\section{A high inflammatory response exacerbates tissue recovery in aged killifish}

263 To be certain that the stab-wound injury created a comparable injury in young and aged animals, 264 we studied the temporal dynamics of the surface size of the injury using (immuno)histology 265 (Figure S2). One hour after injury, the size of the wound was comparable between young adult 266 and aged killifish. By 2 dpi, the injury size was enlarged in aged killifish, possibly due to differences 267 in cell death, arrival of inflammatory cells and edema (Figure 1E). By counting the number of $268 \mathrm{TUNEL}^{+}$apoptotic cells we discovered that the aged, injured brain contained more TUNEL ${ }^{+}$ 269 apoptotic cells than young adult fish already at $1 \mathrm{dpi}(37 \pm 12,33$ versus $15,33 \pm 4,659 ; P=0,0128)$. 270 It thus seems that in an aged injured environment, cells were more vulnerable to secondary 271 damage, which might be linked to an inflammatory state. Led by these results, we stained for the 272 microglia/macrophage marker (pan-leukocyte marker) L-plastin at 1 and 2 dpi. As expected, stab273 wound injury induced an acute inflammatory response reflected in an increased number of 274 microglia/macrophages at 1 and $2 \mathrm{dpi}$ in injured fish compared to naive fish (uninjured controls) 275 (Figure 2). In addition, we detected more microglia/macrophages in aged, injured killifish 276 compared to young adult, injured killifish, indicating a higher inflammatory response in aged 277 killifish (Figure 2). This may explain the higher number of apoptotic cells seen at $1 \mathrm{dpi}$, as well as 
why the size of the injury site increases towards 2 dpi in aged killifish. In addition, a fraction of these microglia/macrophages were $\mathrm{PCNA}^{+}$(Figure S3), and thus proliferating, which is in accordance with reports on injury impact in zebrafish (Kroehne et al., 2011; Baumgart et al., 2012).

\section{Reactive proliferation is declined and delayed in aged injured killifish brains}

283 For brain repair to be successful, new neurons should be generated from the available neuronal 284 progenitor cell (NPC) pool. We counted the number of activated NPCs $\left(\mathrm{SOX}^{+} \mathrm{PCNA}^{+}\right)$and all NPCs $285\left(\mathrm{SOX}^{+}\right)$in the dorsal ventricular zone (VZ) (Figure S4) in function of age and time post injury.

The percentage of dividing NPCs in the progenitor pool of aged naive fish was cleary lower than in young fish $(8,120 \% \pm 1,048$ versus $20,06 \% \pm 3,486 ; P=0,0047$, Figure 3$)$, and this difference persisted upon injury. In young killifish, the injury-induced proliferation of NPCs was significantly higher than in aged killifish (at 2 dpi: 34,94\% $\pm 3,824$ versus 17,75\% $\pm 2,629$ repectively; $P<0,0001$, Figure 3). The increase occured at 1 and $2 \mathrm{dpi}$, and declined back to normal levels from $5 \mathrm{dpi}$ onward. In the aged killifish the percentage was significantly higher at 2 dpi compared to naive aged fish, but it did not even reach the baseline levels of the young adult killifish. These result show that the capacity for NPC proliferation upon injury diminishes steeply with age, but that aged killifish still retain some capacity for NPC reactive proliferation.

\section{Reactive proliferation is mainly supported by NGPs and not RGs}

The killifish dorsal telencephalon holds two classes of progenitors: NGPs and RGs (Coolen et al., 2020). We therefore investigated which progenitor type supported reactive proliferation in our injury model at $2 \mathrm{dpi}$, the time point at which reactive proliferation peaks independent of age (Figure 3). By immunohistochemistry for SOX2, PCNA and BLBP, we could delineate the dividing RGs (SOX2 ${ }^{+} \mathrm{PCNA}^{+} \mathrm{BLBP}^{+}$) from the dividing NGPs (SOX2 ${ }^{+} \mathrm{PCNA}^{+} \mathrm{BLBP}^{-}$). Strikingly, we rarely observed dividing RGs (approximately $3 \%$ of all NPCs) (Figure 4), although RG fibers did present themselves with a swollen morphology after injury (Figure 4B'). Instead, we clearly observed many dividing NGPs in the naive and injured dorsal VZ of young and aged killifish, showing that NGPs are the most prominent cell type supporting adult neurogenesis in killifish (Figure 4). The percentage of dividing NGPs among all NPCs was significantly lower in aged killifish compared to young adult fish (Figure 4 ), both in naive animals $(14,26 \% \pm 1,328$ versus $28,04 \% \pm 1 ; 569 ; P=0,0005)$ and injured animals $(22,5 \% \pm 2,383$ versus $43 \% \pm 2,543 ; \mathrm{P}<0,0001)$, demonstrating that adult neuro(re)genesis declined upon aging.

309 Unlike in zebrafish, we provide evidence that injury-induced reactive proliferation of progenitors 310 is mainly supported by the NGPs in the killifish pallium. Of all dividing NPCs present in the young 311 injured adult VZ at $2 \mathrm{dpi}, 93,49 \% \pm 0,688$ could be assigned to NGPs, while only 6,509\% $\pm 0,688$ 312 were of RG type (Table S1). This is in sharp contrast to young adult injured zebrafish where 
$31386,5 \% \pm 3,1 \%(n=4)$ of dividing cells represented RGs at 3 dpi (Kroehne et al., 2011). In the aged 314 injured killifish $87,72 \% \pm 1,837$ of all dividing NPCs were NGPs, while $12,28 \% \pm 1,837$ were RG (Table

315 S1). Aged injured killifish thus showed a higher percentage of dividing RG among all dividing cells 316 compared to young adult injured killifish. A similar observation was made for naive conditions 317 (Table S1). It appears that aged killifish try to compensate their reduced capacities by activating 318 the RG pool.

319 Taken together, killifish NGPs represent the most potent progenitor type driving the proliferative 320 response to injury, but their number declines significantly with age when RGs appear more 321 activated, albeit still low in repect to NGPs.

\section{A declined number of newborn neurons reach the injury site in aged killifish}

323 To elucidate if reactive NGPs lead to the production of newborn neurons that can migrate to the 324 injury site for replacement of the lost cell types, we designed a BrdU pulse chase experiment. In 325 between 1 and $2 \mathrm{dpi}$, when reactive proliferation is most intense (Figure 3), young and aged 326 injured and age-matched control (AMC) killifish where subjected to 16 hours of BrdU water, 327 labeling all cells passing through S-phase (Figure 5A). After a 21-day chase period, a time window 328 matching maximal recovery in young adult fish, we visualized the progeny of these cells via 329 immunostaining for BrdU and HuCD. HuCD is a pan-neuronal marker, expressed in both early 330 (immature) and late (mature) neurons (Kim et al., 1996; Kroehne et al., 2011). As such, BrdU $331 \mathrm{HuCD}^{+}$neurons, that represent the progeny of the dividing NGPs, were visualized at 23 dpi (Figure $3325 B)$.

333 Our data reveal impaired production and migration of newborn neurons in aged killifish at $23 \mathrm{dpi}$. 334 In naive fish, we could hardly detect newborn neurons that had migrated into the parenchyma $335(10,94 \pm 3,303$ for young adult and 3,571 $\pm 0,566$ for aged fish) (Figure 5C). Many newborn neurons 336 in the periventricular zone (PVZ) of the dorsal pallium and near the rostral migratory stream 337 (RMS) (Figure S4), migrate only 1-2 cell diameters away from the ventricular stem cell zones, 338 which is typical for constitutive neurogenesis in teleosts (Adolf et al., 2006; Grandel et al., 2006; 339 Rothenaigner et al., 2011). Injured killifish on the contrary, generated more newborn neurons 340 that migrated into the injured parenchyma. This number was significantly higher in young adult 341 killifish than in aged killifish $(46,97 \pm 8,017$ versus $20,80 \pm 6,540 ; P=0,005)$ (Figure $5 C$ ).

342 Most likely the impaired replenishment of newborn neurons in aged injured animals is caused by 343 a declined production of neurons, yet also the failure of these neurons to migrate towards the 344 injury site through the aged non-permissive environment. Indeed, we confirmed that aged 345 injured killifish produce large numbers of newborn microglia/macrophages (BrdU ${ }^{+}$L-plastin $^{+}$ 346 cells) by 23 dpi (aged killifish: $30,33 \pm 5,88$ versus young adult killifish: 4,933 $\pm 1,087 ; P<0,0001$ ), 347 what most likely contributes to an inflammatory non-permissive environment (Figure 5D,E). 


\section{Aging hampers the replenishment of progenitors in the VZ after injury}

Stab-wound injury disrupts the parenchyma of the dorsal telencephalon, but also the VZ. Hence, the dorsal VZ needs to be replenished with newly generated NGPs and RGs. We applied BrdU pulse chase labeling to investigate if dividing progenitors, labeled between 1 and $2 \mathrm{dpi}$, give rise to new progenitors by $23 \mathrm{dpi}$, the time point where regeneration is complete in young adult fish (Figure 6A). We zoomed in on the two major progenitor classes; (1) newly generated RGs (BrdU', $\mathrm{BLBP}^{+}$) and (2) newly generated dividing NGPs (BrdU $\left.{ }^{+}, \mathrm{PCNA}^{+}, \mathrm{BLBP}^{-}\right)$. The BrdU signal in highly proliferative progenitors, which are NGPs, will be diluted with each cell division until the moment when the BrdU signal gets lost. This assay thus rather describes the progeny of low proliferative progenitors, that is the RGs in killifish. Indeed, in all conditions we found a larger number of newly generated RGs than NGPs. RGs, labeled between 1 and 2 dpi, thus seem to generate new RGs via gliogenic divisions. Whether RGs also gave rise to NGPs or vice versa remains elusive and an interesting research question for future studies. Of note, we also detected a low number of $\mathrm{BrdU}^{+}$ cells in the VZ that were PCNA- and BLBP-, which we thus could not identify as RG or NGP. We however realize that these cells could possibly represent a quiescent progenitor type or newborn neuroblasts, lying closely to the VZ.

Independent of the progenitor class, we observed that naive fish showed negligible amounts of newborn RG and NGPs. In injured brains, clearly more newly generated progenitors are present in the dorsal VZ, albeit much less in aged fish compared to young adult fish (for newly generated versus $3,3 \pm 0,5925 ; P=$ n.s.) (Figure $6 B, C$ ).

Remarkable, the dividing progenitor pool was replenished to baseline levels during the 21 day chase for each specific age (Figure S5). For both progenitor classes, a similar number of dividing cells was observed compared to their respective AMCs (Figure S5). Aged fish however still had a

372 lower number of dividing NGPs at 23 dpi compared to young adult animals. The number of 373 dividing RGs was very low for both ages.

374 Taken together, these results indicate that aged killifish are less efficient in replenishing the 375 dorsal VZ after stab-wound injury compared to young adult killifish. Furthermore, generation of 376 neurons and progenitors does not exhaust the available dividing progenitor pools during a short 37721 day chase period, suggesting a great capacity of killifish for progenitor cell self-renewal and neuro(re)genesis. This capacity was again higher in young adult killifish.

\section{Discussion}

380 We designed and benchmarked an easy-to-use and adequate TBI model to study 381 neuroregeneration and uncover the impact of aging on brain repair in the fastest-aging teleost 382 laboratory species, $N$. furzeri. We discovered an age-related decline in proliferation as well as 
injury-related proliferative response of neurogenic progenitors. The NGP population is most active and responds most prominently to injury. Glial scarring and a strong, localized inflammatory reaction also typify the aged condition post-injury, while young adult brains regenerate in a seamless manner. Taken together, our results provide a validated animal model for future studies to unveil underlying mechanisms driving the loss of full neuroregenerative capacity upon aging.

Stab-wound injuries are reliable, effective injury models and require no specialized equipment, which makes them applicable in a broad range of laboratories. They are also very practical to study a vast array of pathological conditions, since they elicit a clear multicellular read-out, e.g. disruption of the blood brain barrier (BBB), inflammation, astrogliosis. Stab-injuries are often used in mice as TBI model, e.g. Balasingam et al., 1994; Allahyari et al., 2015; Frik et al., 2018; Hashimoto et al., 2018; Mattugini et al., 2018. They have also been extensively characterized in the zebrafish telencephalon (Ayari et al., 2010; Kroehne et al., 2011; März et al., 2011; Baumgart et al., 2012; Kishimoto et al., 2012; Kyritsis et al., 2012; Barbosa et al., 2015). The benefit of translating this model to the killifish is the implementation of the factor age to investigate its influence on the high neuroregenerative capacity, typically associated with teleost species. Surgery can be performed under 5 minutes, no specialized equipment is required and since teleost fish are regenerative-competent vertebrates, full recovery is established within 23-30 days in young adult killifish (data presented here) and adult zebrafish (Ayari et al., 2010; Kroehne et al., 2011; Kishimoto et al., 2012).

The current study provides evidence that the killifish telencephalon is subjected to ageassociated stem cell exhaustion, one of the 9 hallmarks of aging (López-Otín et al., 2013). Farr less proliferating progenitors, newborn neurons and newly generated progenitors were counted in aged killifish, even after injury, which is in line with other reports in killifish (Tozzini et al., 2012) and zebrafish (Edelmann et al., 2013; Bhattarai et al., 2017). The most striking difference to other teleosts is that the reactive proliferation is related to the atypical NGPs in killifish, instead of the typical RGs that support zebrafish neuroregeneration (Kroehne et al., 2011; Baumgart et al., 2012). RG division is very low in killifish, even after injury. It was recently discovered that RGs enter a Notch3-dependent quiescence already in larval stages (Coolen et al., 2020). Whenever these RGs are reactivated, their division is mainly of gliogenic nature (Coolen et al., 2020). The low division of RGs in the killifish brain is reminiscent of adult neural stem cells (NSCs) in the SGZ and SVZ of mice. Here, GFAP ${ }^{+}$NSCs are mostly quiescent and use predominately asymmetric divisions to create a new NSC and a transient amplifying progenitor. They can however also use symmetric divisions for self-renewal (reviewed in Daynac et al., 2017). Indeed, we and others discovered that killifish RGs, are mostly quiescent, have low division potential and can also selfrenew (Coolen et al., 2020). Whether killifish RGs can also generate NGPs via asymmetric division the way mammalian NSCs create transit amplifying cells remains however elusive. There are 
420 some similarities between NGPs and mammalian transient amplifying progenitors. Both 421 progenitor types have a higher division rate compared to NSCs/RGs and are responsible for the 422 production of newborn neurons (neuroblasts) (Daynac et al., 2017; Coolen et al., 2020). In the 423 SVZ, generation of neurons happen via symmetric divisions, directly creating two neuroblasts 424 from one transit amplifying progenitor (Daynac et al., 2017). NGPs on the contrary, also appear 425 to have a lot of self-renewing capacity (Coolen et al., 2020). As such, NGPs might represent a 426 separate progenitor lineage, created early in brain development, that is self-sustainable while 427 the RGs become quiescent upon adulthood. In the present study we also discovered that the 428 percentage of dividing RGs among all dividing cells was doubled in aged killifish, although still low 429 in respect to the percentage of dividing NGPs. We hypothesise that when NGPs become 430 exhausted with increasing age, more RGs become activated as a replenishing strategy. This would 431 imply that RGs give rise to NGPs, which remains unexplored to date. Taken together, our results 432 urge for dedicated lineage tracing from these two progenitor pools. Insights into the self-renewal 433 capacities of both progenitor types might unravel key concepts of stem cell biology and division 434 mode necessary for successful neuroregeneration.

435 Notwithstanding the low percentage of dividing progenitors, aged killifish are still able to produce 436 a neurogenic response upon injury. They generate a considerable number of newborn neurons, 437 albeit much less than young adult killifish. Few neurons reach the injury site in the parenchyma, 438 suggesting migration is also impacted by aging. It remains to be investigated if these neurons are 439 still capable of maturing and integrating in the existing aged circuit, and if some degree of 440 improved functional outcome can be established. The massive surge in microglia/macrophages 441 at the injury site in aged killifish, likely creates chronic inflammation and renders the parenchyma 442 unsuitable for neuron migration, maturation and integration, or may even drive the newborn 443 neurons into apoptosis. This is similar to mammals, in which a large portion of the injury-induced 444 neurons will die due to the pathological environment (Turnley et al., 2014; Grade et al., 2017). 445 We predict the chronic inflammation to be partly caused by influx of blood-derived macrophages. 446 Aging renders the BBB weak, enabling more macrophages to pass the BBB in aged brains 447 (Montagne et al., 2015; Verheggen et al., 2020). This will increase the inflammatory response and 448 exacerbate damage. Also in the aged zebrafish telencephalon, increased numbers of ramified, 449 but not round, microglia were discovered after amyloidosis (Bhattarai et al., 2017).

450 Aged killifish also showed glial scarring at the injury site, which is - to our knowledge - never 451 whitnessed before in other regeneration studies working with telencephalic injuries in teleosts 452 (Kroehne et al., 2011; Baumgart et al., 2012; Edelmann et al., 2013; Bhattarai et al., 2017). In 453 mammals, the glial scar acts as a physical barrier consisting of reactive astrocytes, NG2 glia and 454 microglia. On the one hand, the glial scar preserves tissue integrity by repairing the BBB and 455 blocking the influx of fibrotic cells and blood-derived macrophages. On the other hand, the scar 456 represents a physical barrier for axonal outgrowth, thereby restricting neural circuit integration 
457 and overall brain repair (reviewed in Adams et al., 2018). We hypothesize that also in aged injured 458 killifish, the glial scar is preventing newborn neurons to integrate and thereby impedes successful 459 brain recovery. Interestingly, the presence of RG fibers, expressing high amounts of GS, suggests 460 a function of RGs in glial scar formation. GS is known to convert glutamate into glutamine, 461 reducing toxic extracellular glutamate levels (Zou et al., 2010). Its presence at the glial scar could 462 indicate that high glutamate toxicity levels were still present in aged killifish. The cells 463 surrounding the scar tissue indeed had a swollen morphology, typical for cytotoxicity (Liang et 464 al., 2007).

465 Since our data predict that RGs do not support the production of new neurons in the killifish 466 brain, the question remains what role RGs play after injury. At 2 dpi, RG fibers appeared swollen 467 in aged injured killifish, suggesting that the RGs do respond to the insult. Considering that many 468 of the mammalian astrocyte-specific genes (GLT-1, BLBP, GS, GLAST, aldh1l1, GFAP, Vimentin, $469 \mathrm{~S} 100 \beta$ ) are also expressed by teleost RGs (Ganz et al., 2010; März et al., 2010; Chen et al., 2020; 470 Coolen et al., 2020), a similar function is highly likely. In mammals, RGs act as neural stem cells 471 early in development and support neurogenesis. Later on, most RGs leave the ventricular zone 472 the mammalian stem cell zone - and become star-shaped astrocytes (reviewed in Kriegstein et 473 al., 2009). 'Star-shaped astroglia' have previously been described in the zebrafish spinal cord 474 (Kawai et al., 2001), but are are yet to be found in the teleost telencephalon. New evidence was 475 recently provided that teleost 'astroglia' resemble mammalian astrocytes more than once 476 thought. In the larval zebrafish spinal cord they are in close association with synapses, exhibit 477 tiling and calcium signaling dynamics and have a long bushy morphology that expands during 478 development (Chen et al., 2020). In the zebrafish larval brain, interplay between astroglia and 479 neurons during epileptic seizures was discovered, as well as large gap junction-coupled glial 480 networks (Diaz Verdugo et al., 2019). It thus seems that teleost RGs keep their developmental 481 morphology into adulthood, but adopt several functions typically associated with mammalian 482 protoplasmic astrocytes (Wahis et al., 2021). This might also explain why teleosts have such 483 impressive regenerative abilities as their astroglia resemble more the 'developmental' 484 mammalian RGs, which makes them highly neurogenic into adulthood. The killifish thus 485 represents a promising vertebrate model to unravel the function of the teleost 'astroglia' because 486 the delineation between RG stem cell properties and astrocyte-properties seems more 487 distinguished than in other teleost models. How these killifish 'astroglia' respond to injury and, 488 interestingly, behave upon brain aging, are intriguing research questions for the future.

489 Altogether, we expose the aged killifish to model low regenerative abilities similar to adult 490 mammals. The aged neuroregenerative response is characterized by glial scarring, secondary 491 damage, aggravating inflammation, reduced proliferation of stem cells and reduced production 492 of new neurons. Our model will therefore be highly useful to elucidate how to reverse the aging 493 state of the brain in order to reinstate a high neuroreparative strategy as occruing in young adult 
494 killifish. Such insights will hopefully play a pivotal role in boosting neurorepair in mammals in the 495 near future.

496 Author Contributions

497 J.V.H.: Conceptualization, design, experiments, statistical analysis, writing, original draft, review, 498 editing and visualization. V.M.: Experiments, statistical analysis, review and editing. S.V.: 499 Experiments. C.Z., L.M., R.A., E.S.: Review and editing. L.A.: Conceptualization, design, writing, 500 review, editing, study supervision.

\section{Funding}

502 This work was supported by Fonds voor Wetenschappelijk Onderzoek (FWO Vlaanderen) 503 research grant number G0C2618N and a personal fellowship to JVH (1S00318N) and SV $504(1 \mathrm{~S} 16617 \mathrm{~N})$. Killifish housing and breeding was supported by a KU Leuven equipment grant (KA505 16-00745).

\section{Declaration of Interest}

507 The authors declare no competing interest.

508 List of abbreviations

509 AMC: age-matched control

510 BBB: brain blood barrier

511 BLBP: brain lipid-binding protein

512 CNS: central nervous system

513 dpi: days post injury

514 GFAP: glial fibrillary acidic protein

515 GS: glutamine synthetase

516 hpi: hours post injury

517 NGP: non-glial progenitor

518 NPC: neuronal progenitor cell

519 NSC: neuronal stem cell

520 PCNA: proliferating cell nuclear antigen

521 PVZ: periventricular zone

522 RG: radial glia

523 RMS: rostral migratory stream

524 SGZ- subgranular zone

525 SOX2: SRY-box transcription factor 2

526 SVZ: subventicular zone

527 TBI: traumatic brain injury 
528 TUNEL: terminal deoxynucleotidyl transferase-mediated dUTP nick end labeling

529 VZ: ventricular zone

530 Acknowledgments

531 We thank our collaborators of the KU Leuven killifish consortium Prof. Dr. L. Brendonck and Dr.

532 T. Pinceel to provide African turquoise killifish eggs of the short-lived GRZ-AD strain. We are

533 grateful to Dr. T. Pinceel for his excellent problem-solving skills in relation to killifish breeding and

534 housing. We thank Rony Van Aerschot, Arnold Van Den Eynde, and the KU Leuven killifish team

535 for taking care of the animals and setting up our shared fish facility. We are thankful to Ria

536 Vanlaer and Lieve Geenen for their technical support. Students that were involved in optimizing

537 techniques are Niels Vidal, Karen Libberecht and Lotte De Bauw. We are also grateful to Dr. Jessie

538 Van houcke, Dr. S. Gilissen and Dr. M. Hennes for reviewing this manuscript. We acknowledge

539 Biorender.com for delivering the tools to create Figure S4.

\section{References}

541 Adams, K. L. and Gallo, V. (2018) The diversity and disparity of the glial scar, Nature Neuroscience, 542 21(1), pp. 9-15. doi: 10.1038/s41593-017-0033-9.

543 Adolf, B., Chapouton, P., Lam, C. S., Topp, S., Tannhäuser, B., Strähle, U., et al. (2006) Conserved

544 and acquired features of adult neurogenesis in the zebrafish telencephalon, Developmental 545 Biology, 295(1), pp. 278-293. doi: 10.1016/j.ydbio.2006.03.023.

546 Allahyari, R. V. and Garcia, A. D. R. (2015) Triggering Reactive Gliosis In Vivo by a Forebrain Stab 547 Injury, Journal of Visualized Experiments, (100), p. e52825. doi: 10.3791/52825.

548 Arvidsson, A., Collin, T., Kirik, D., Kokaia, Z. and Lindvall, O. (2002) Neuronal replacement from 549 endogenous precursors in the adult brain after stroke, Nature Medicine, 8(9), pp. 963-70. doi: $55010.1038 / \mathrm{nm} 747$.

551 Ayari, B., El Hachimi, K. H., Yanicostas, C., Landoulsi, A. and Soussi-Yanicostas, N. (2010) 552 Prokineticin 2 expression is associated with neural repair of injured adult zebrafish 553 telencephalon, Journal of Neurotrauma, 27(5), pp. 959-72. doi: 10.1089/neu.2009.0972.

554 Balasingam, V., Tejada-Berges, T., Wright, E., Bouckova, R. and Yong, V. (1994) Reactive 555 astrogliosis in the neonatal mouse brain and its modulation by cytokines, The Journal of 556 Neuroscience, 14(2), pp. 846-856. doi: 10.1523/JNEUROSCI.14-02-00846.1994.

557 Barbosa, J. S., Sanchez-Gonzalez, R., Di Giaimo, R., Baumgart, E. V., Theis, F. J., Götz, M., et al. 558 (2015) Live imaging of adult neural stem cell behavior in the intact and injured zebrafish brain, 559 Science, 348(6236), pp. 789-793. doi: 10.1126/science.aaa2729.

560 Baumgart, E. V, Barbosa, J. S., Bally-Cuif, L., Gotz, M. and Ninkovic, J. (2012) Stab wound injury of 561 the zebrafish telencephalon: A model for comparative analysis of reactive gliosis, Glia, 60(3), pp.

562 343-357. doi: 10.1002/glia.22269. 
Bhattarai, P., Thomas, A. K., Zhang, Y. and Kizil, C. (2017) The effects of aging on Amyloid-beta42induced neurodegeneration and regeneration in adult zebrafish brain, Neurogenesis (Austin), 4(1), p. e1322666. doi: 10.1080/23262133.2017.1322666.

Chen, J., Poskanzer, K. E., Freeman, M. R. and Monk, K. R. (2020) Live-imaging of astrocyte morphogenesis and function in zebrafish neural circuits, Nature Neuroscience, 23, pp. 12971306. doi: 10.1038/s41593-020-0703-x.

Coolen, M., Labusch, M., Mannioui, A. and Bally-Cuif, L. (2020) Mosaic Heterochrony in Neural Progenitors Sustains Accelerated Brain Growth and Neurogenesis in the Juvenile Killifish N. furzeri, Current Biology, 30(4), pp. 736-745.e4. doi: 10.1016/j.cub.2019.12.046.

572 Daynac, M. and Petritsch, C. K. (2017) Regulation of Asymmetric Cell Division in Mammalian Neural Stem and Cancer Precursor Cells, in Tassan, J. P. and Kubiak, J. (eds) Asymmetric Cell Division in Development, Differentiation and Cancer. Results and Problems in Cell Differentiation. vol. 61. Springer, Cham, pp. 375-399. doi: 10.1007/978-3-319-53150-2_17.

Diaz Verdugo, C., Myren-Svelstad, S., Aydin, E., Van Hoeymissen, E., Deneubourg, C., seizures, Nature Communications, 10(3830), pp. 1-13. doi: 10.1038/s41467-019-11739-z.

579 Ding, L., Kuhne, W. W., Hinton, D. E., Song, J. and Dynan, W. S. (2010) Quantifiable biomarkers of normal aging in the Japanese Medaka fish (Oryzias latipes), PLOS ONE, 5(10), p. e13287. doi: 10.1371/journal.pone.0013287.

582 Dugger, B. N. and Dickson, D. W. (2017) Pathology of Neurodegenerative Diseases, Cold Spring Harbor Perspectives in Biology, 9(7), p. a028035. doi: 10.1101/cshperspect.a028035.

584 Edelmann, K., Glashauser, L., Sprungala, S., Hesl, B., Fritschle, M., Ninkovic, J., et al. (2013) Increased radial glia quiescence, decreased reactivation upon injury and unaltered neuroblast behavior underlie decreased neurogenesis in the aging zebrafish telencephalon, J Comp Neurol, 521(13), pp. 3099-3115. doi: 10.1002/cne.23347. the Iceberg: Assessing the Global Socioeconomic Costs of Alzheimer's Disease and Related Dementias and Strategic Implications for Stakeholders, Journal of Alzheimer's Disease, 70(2), pp. 323-341. doi: 10.3233/JAD-190426. reports, 19(5). doi: 10.15252/embr.201745294.

595 Galvan, V. and Jin, K. (2007) Neurogenesis in the aging brain., Clinical interventions in aging, 2(4), 596 pp. 605-610. doi: 10.2147/cia.s1614.

597 Ganz, J., Kaslin, J., Hochmann, S., Freudenreich, D. and Brand, M. (2010) Heterogeneity and Fgf 598 dependence of adult neural progenitors in the zebrafish telencephalon, GLIA, 58(11), pp. 1345599 63. doi: 10.1002/glia.21012. 
600 Genade, T., Benedetti, M., Terzibasi, E., Roncaglia, P., Valenzano, D. R., Cattaneo, A., et al. (2005) 601 Annual fishes of the genus Nothobranchius as a model system for aging research, Aging Cell, 4(5), 602 pp. 223-233. doi: 10.1111/j.1474-9726.2005.00165.x.

603 Gerhard, G. S., Kauffman, E. J., Wang, X., Stewart, R., Moore, J. L., Kasales, C. J., et al. (2002) Life spans and senescent phenotypes in two strains of Zebrafish (Danio rerio), Experimental Gerontology, 37(8-9), pp. 1055-68. doi: 10.1016/S0531-5565(02)00088-8. longevity gender gap, a natural drop in estrogen and telomere shortening during aging: A unique model for studying sex-dependent longevity, Frontiers in Zoology, 10(1), p. 78. doi: 10.1186/1742-9994-10-78.

610 Grade, S. and Götz, M. (2017) Neuronal replacement therapy: previous achievements and challenges ahead, npj Regenerative Medicine, 2, p. 29. doi: 10.1038/s41536-017-0033-0.

612 Grandel, H., Kaslin, J., Ganz, J., Wenzel, I. and Brand, M. (2006) Neural stem cells and 613 neurogenesis in the adult zebrafish brain: Origin, proliferation dynamics, migration and cell fate, Developmental Biology, 295(1), pp. 263-277. doi: 10.1016/j.ydbio.2006.03.040. of Comparative Neurology. doi: 10.1002/cne.22481.

617 Hashimoto, K., Nakashima, M., Hamano, A., Gotoh, M., Ikeshima-Kataoka, H., MurakamiMurofushi, K., et al. (2018) 2-carba cyclic phosphatidic acid suppresses inflammation via regulation of microglial polarisation in the stab-wounded mouse cerebral cortex, Scientific Reports, 8(1), p. 9715. doi: 10.1038/s41598-018-27990-1.

621 Hou, Y., Dan, X., Babbar, M., Wei, Y., Hasselbalch, S. G., Croteau, D. L., et al. (2019) Ageing as a 622 risk factor for neurodegenerative disease, Nature Reviews Neurology, 15(10), pp. 565-581. doi: 10.1038/s41582-019-0244-7.

624 Howe, K., Clark, M. D., Torroja, C. F., Torrance, J., Berthelot, C., Muffato, M., et al. (2013) The 625 zebrafish reference genome sequence and its relationship to the human genome, Nature. 626 2013/04/19, 496(7446), pp. 498-503. doi: 10.1038/nature12111.

$627 \mathrm{Hu}, \mathrm{C} .-\mathrm{K}$. and Brunet, A. (2018) The African turquoise killifish: A research organism to study 628 vertebrate aging and diapause, Aging Cell, 17(3), p. e12757. doi: 10.1111/acel.12757.

629 Kawai, H., Arata, N. and Nakayasu, H. (2001) Three-dimensional distribution of astrocytes in 630 zebrafish spinal cord, GLIA, 36, pp. 406-413. doi: 10.1002/glia.1126.

631 Kernie, S. G. and Parent, J. M. (2010) Forebrain neurogenesis after focal Ischemic and traumatic 632 brain injury, Neurobiol Dis. 2009/11/17, 37(2), pp. 267-274. doi: 10.1016/j.nbd.2009.11.002.

633 Kim, C. H., Ueshima, E., Muraoka, O., Tanaka, H., Yeo, S. Y., Huh, T. L., et al. (1996) Zebrafish 634 elav/HuC homologue as a very early neuronal marker, Neuroscience Letters, 216(2), pp. 109-112. 635 doi: 10.1016/0304-3940(96)13021-4.

636 Kim, Y., Nam, H. G. and Valenzano, D. R. (2016) The short-lived African turquoise killifish: An 
637 emerging experimental model for ageing, Disease Models and Mechanisms, 9(2), pp. 115-129.

638 Kishimoto, N., Shimizu, K. and Sawamoto, K. (2012) Neuronal regeneration in a zebrafish model 639 of adult brain injury, Dis Model Mech. 2011/10/27, 5(2), pp. 200-209. doi: 10.1242/dmm.007336.

640 Kriegstein, A. and Alvarez-Buylla, A. (2009) The Glial Nature of Embryonic and Adult Neural Stem

641 Cells, Annual Review of Neuroscience, 32(1), pp. 149-184. doi:

642 10.1146/annurev.neuro.051508.135600.

643 Kroehne, V., Freudenreich, D., Hans, S., Kaslin, J. and Brand, M. (2011) Regeneration of the adult 644 zebrafish brain from neurogenic radial glia-type progenitors, Development, 138(22), pp. 4831645 4841. doi: 10.1242/dev.072587.

646 Kyritsis, N., Kizil, C., Zocher, S., Kroehne, V., Kaslin, J., Freudenreich, D., et al. (2012) Acute 647 inflammation initiates the regenerative response in the adult zebrafish brain, Science, 338(6112), 648 pp. 1353-6. doi: 10.1126/science.1228773.

649 Liang, D., Bhatta, S., Gerzanich, V. and Simard, J. M. (2007) Cytotoxic edema: mechanisms of 650 pathological cell swelling, Neurosurgical Focus, 22(5), pp. 1-9. doi: 10.3171/foc.2007.22.5.3.

651 López-Otín, C., Blasco, M. A., Partridge, L., Serrano, M. and Kroemer, G. (2013) The Hallmarks of 652 Aging, Cell, 153(6), pp. 1194-1217. doi: 10.1016/j.cell.2013.05.039.

653 Marques, I. J., Lupi, E. and Mercader, N. (2019) Model systems for regeneration: Zebrafish, 654 Development (Cambridge), 146(18), pp. 1-13. doi: 10.1242/dev.167692.

März, M., Chapouton, P., Diotel, N., Vaillant, C., Hesl, B., Takamiya, M., et al. (2010) Heterogeneity in progenitor cell subtypes in the ventricular zone of the zebrafish adult telencephalon, GLIA, 58(7), pp. 870-88. doi: 10.1002/glia.20971.

658 März, M., Schmidt, R., Rastegar, S. and Strahle, U. (2011) Regenerative response following stab injury in the adult zebrafish telencephalon, Developmental Dynamics, 240(9), pp. 2221-31. doi: $10.1002 / d v d y .22710$.

661 Mattugini, N., Merl-Pham, J., Petrozziello, E., Schindler, L., Bernhagen, J., Hauck, S. M., et al. (2018) Influence of white matter injury on gray matter reactive gliosis upon stab wound in the adult murine cerebral cortex, Glia, 66(8), pp. 1644-1662. doi: 10.1002/glia.23329. and delayed maturation in wild-derived mice, Experimental Biology and Medicine, 227(7), pp. 500-8. doi: 10.1177/153537020222700715.

672 Mueller, T. and Wullimann, M. F. (2009) An evolutionary interpretation of teleostean forebrain 
anatomy, in Brain, Behavior and Evolution. doi: 10.1159/000229011.

674 Platzer, M. and Englert, C. (2016) Nothobranchius furzeri: A Model for Aging Research and More,

675 Trends Genet. 2016/07/19, 32(9), pp. 543-552. doi: 10.1016/j.tig.2016.06.006.

676 Polačik, M., Blažek, R. and Reichard, M. (2016) Laboratory breeding of the short-lived annual

677 killifish Nothobranchius furzeri, Nature Protocols, 11, pp. 1396-1413. doi:

678 10.1038/nprot.2016.080.

679 Popa-Wagner, A., Buga, A. M. and Kokaia, Z. (2011) Perturbed cellular response to brain injury 680 during aging, Ageing Research Reviews. doi: 10.1016/j.arr.2009.10.008.

681 Reichwald, K., Petzold, A., Koch, P., Downie, B. r, Hartmann, N., Pietsch, S., et al. (2015) Insights 682 into Sex Chromosome Evolution and Aging from the Genome of a Short-Lived Fish, Cell, 163(6), 683 pp. 1527-1538. doi: 10.1016/j.cell.2015.10.071.

684 Rothenaigner, I., Krecsmarik, M., Hayes, J. A., Bahn, B., Lepier, A., Fortin, G., et al. (2011) Clonal analysis by distinct viral vectors identifies bona fide neural stem cells in the adult zebrafish telencephalon and characterizes their division properties and fate, Development, 138(8), pp. 1459-1469. doi: 10.1242/dev.058156.

688 Tanaka, E. M. and Ferretti, P. (2009) Considering the evolution of regeneration in the central nervous system, Nature Reviews Neuroscience, 10(10), pp. 713-723. doi: 10.1038/nrn2707.

690 Thored, P., Arvidsson, A., Cacci, E., Ahlenius, H., Kallur, T., Darsalia, V., et al. (2006) Persistent 691 Production of Neurons from Adult Brain Stem Cells During Recovery after Stroke, Stem Cells, 692 24(3), pp. 739-747. doi: 10.1634/stemcells.2005-0281.

693 Tozzini, E. T., Baumgart, M., Battistoni, G. and Cellerino, A. (2012) Adult neurogenesis in the short694 lived teleost Nothobranchius furzeri: localization of neurogenic niches, molecular 695 characterization and effects of aging, Aging Cell, 11(2), pp. 241-251. doi: 10.1111/j.1474696 9726.2011.00781.x.

697 Turnley, A. M., Basrai, H. S. and Christie, K. J. (2014) Is integration and survival of newborn 698 neurons the bottleneck for effective neural repair by endogenous neural precursor cells?, 699 Frontiers in Neuroscience, 8. doi: 10.3389/fnins.2014.00029.

700 Valdesalici, S. and Cellerino, A. (2003) Extremely short lifespan in the annual fish Nothobranchius 701 furzeri, Proceedings of the Royal Society of London. Series B: Biological Sciences, 270(suppl_2), 702 pp. S189-S191. doi: 10.1098/rsbl.2003.0048.

703 Valenzano, D. R., Benayoun, B. A., Singh, P. P., Zhang, E., Etter, P. D., Hu, C.-K., et al. (2015) The African Turquoise Killifish Genome Provides Insights into Evolution and Genetic Architecture of Lifespan., Cell, 163(6), pp. 1539-1554. doi: 10.1016/j.cell.2015.11.008.

706 Van houcke, J., De Groef, L., Dekeyster, E. and Moons, L. (2015) The zebrafish as a gerontology 707 model in nervous system aging, disease, and repair, Ageing Research Reviews, 24(Pt B), pp. 358708 368. doi: 10.1016/j.arr.2015.10.004.

709 Van houcke, J., Mariën, V., Zandecki, C., Seuntjens, E., Ayana, R. and Arckens, L. (2021) Modeling 
neuroregeneration and neurorepair in an aging context: the power of a teleost model, Front. Cell

713 Verheggen, I. C. M., de Jong, J. J. A., van Boxtel, M. P. J., Gronenschild, E. H. B. M., Palm, W. M.,

714 Postma, A. A., et al. (2020) Increase in blood-brain barrier leakage in healthy, older adults, 715 GeroScience. doi: 10.1007/s11357-020-00211-2.

716 Wahis, J., Hennes, M., Arckens, L. and Holt, M. G. (2021) Star power: the emerging role of 717 astrocytes as neuronal partners during cortical plasticity, Current Opinion in Neurobiology, 67, 718 pp. 174-182. doi: 10.1016/j.conb.2020.12.001.

719 Wendler, S., Hartmann, N., Hoppe, B. and Englert, C. (2015) Age-dependent decline in fin 720 regenerative capacity in the short-lived fish Nothobranchius furzeri, Aging Cell, 14(5), pp. 857721 866. doi: 10.1111/acel.12367.

722 Zambusi, A. and Ninkovic, J. (2020) Regeneration of the central nervous system-principles from 723 brain regeneration in adult zebrafish, World Journal of Stem Cells, 12(1), pp. 8-24. doi: 724 10.4252/wjsc.v12.i1.8.

725 Zhao, A., Qin, H. and Fu, X. (2016) What determines the regenerative capacity in animals?, 726 BioScience, 66(9), pp. 735-746. doi: 10.1093/biosci/biw079.

727 Zou, J., Wang, Y.-X., Dou, F.-F., Lü, H.-Z., Ma, Z.-W., Lu, P.-H., et al. (2010) Glutamine synthetase 728 down-regulation reduces astrocyte protection against glutamate excitotoxicity to neurons, 729 Neurochemistry International, 56(4), pp. 577-584. doi: 10.1016/j.neuint.2009.12.021.

730 Zupanc, G. K. H. (2001) Adult Neurogenesis and Neuronal Regeneration in the Central Nervous 731 System of Teleost Fish, Brain, Behavior and Evolution, 58(5), pp. 250-275. doi: $73210.1159 / 000057569$.

733 Zupanc, G. K. H. and Sîrbulescu, R. F. (2012) Teleost Fish as a Model System to Study Successful 734 Regeneration of the Central Nervous System, in Heber-Katz, E. and Stocum, D. (eds) New 735 Perspectives in Regeneration. Current Topics in Microbiology and Immunology. vol 367. Springer, 736 Berlin, Heidelberg, pp. 193-233. doi: 10.1007/82_2012_297. 
bioRxiv preprint doi: https://doi.org/10.1101/2021.02.26.433041 this version posted February 27, 2021. The copyright holder for this preprint (which was not certified by peer review) is the author/funder, who has granted bioRxiv a license to display the preprint in perpetuity. It is made available under aCC-BY-NC-ND 4.0 International license.

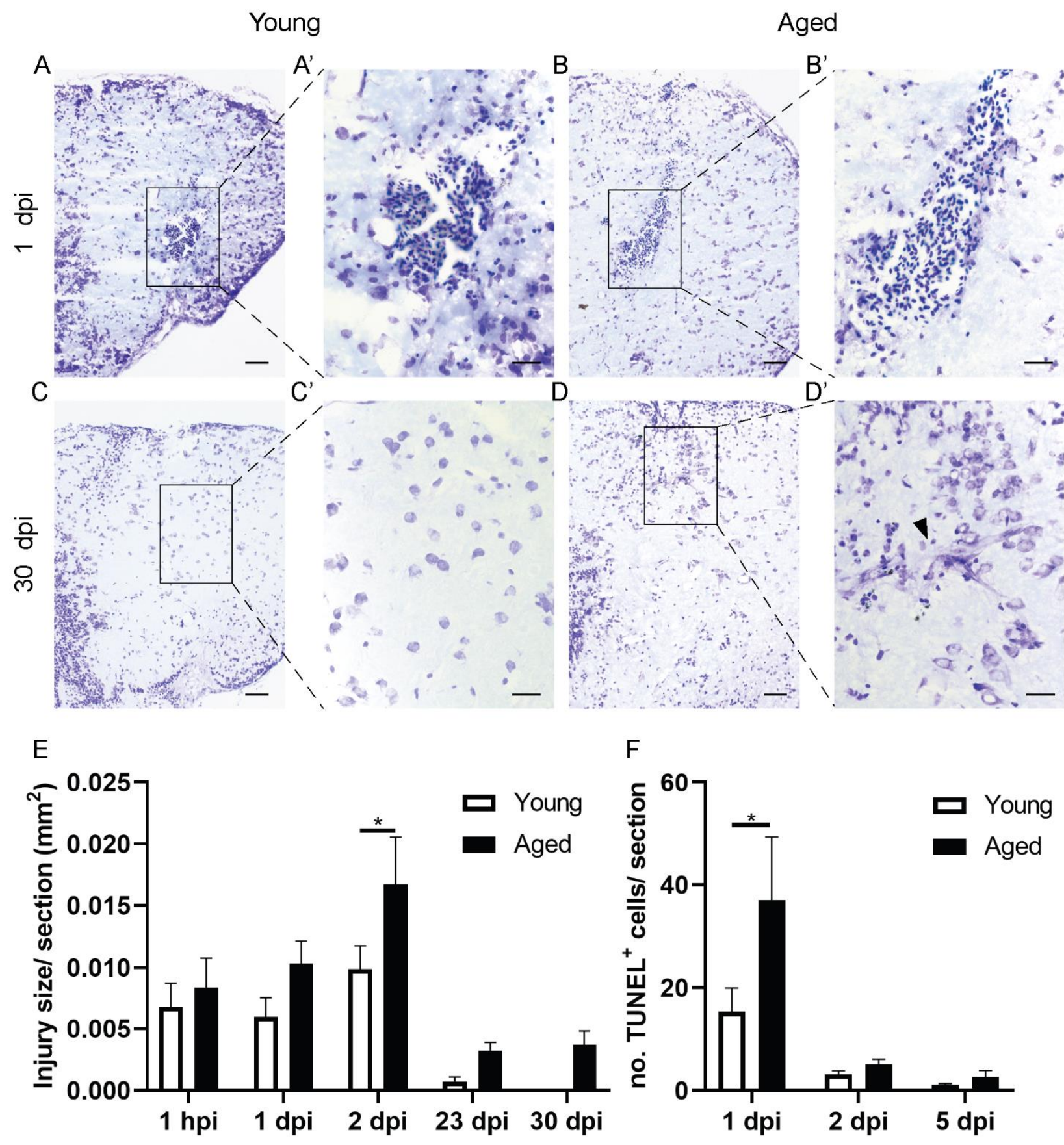

738 Figure 1 


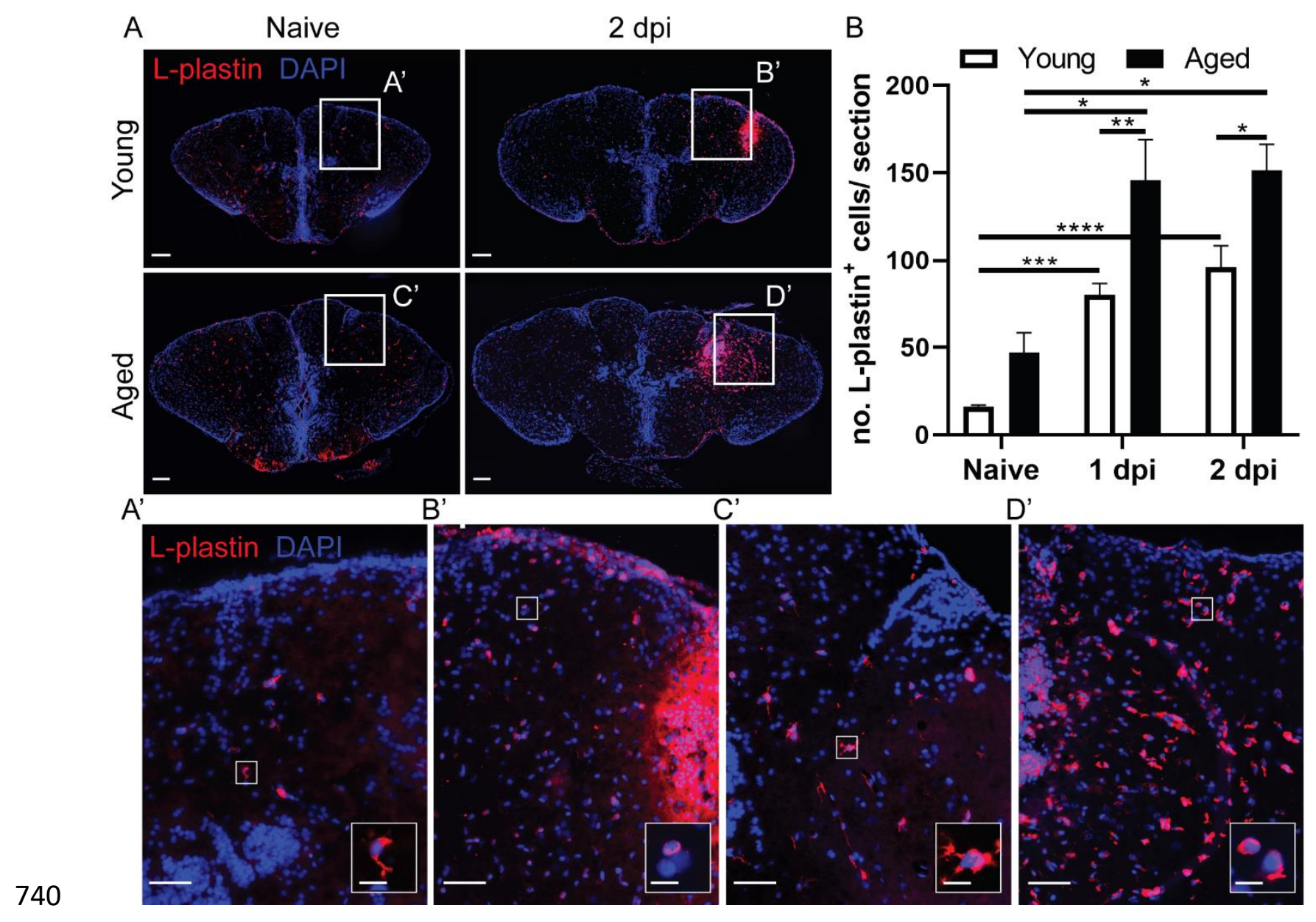

$741 \quad$ Figure 2 

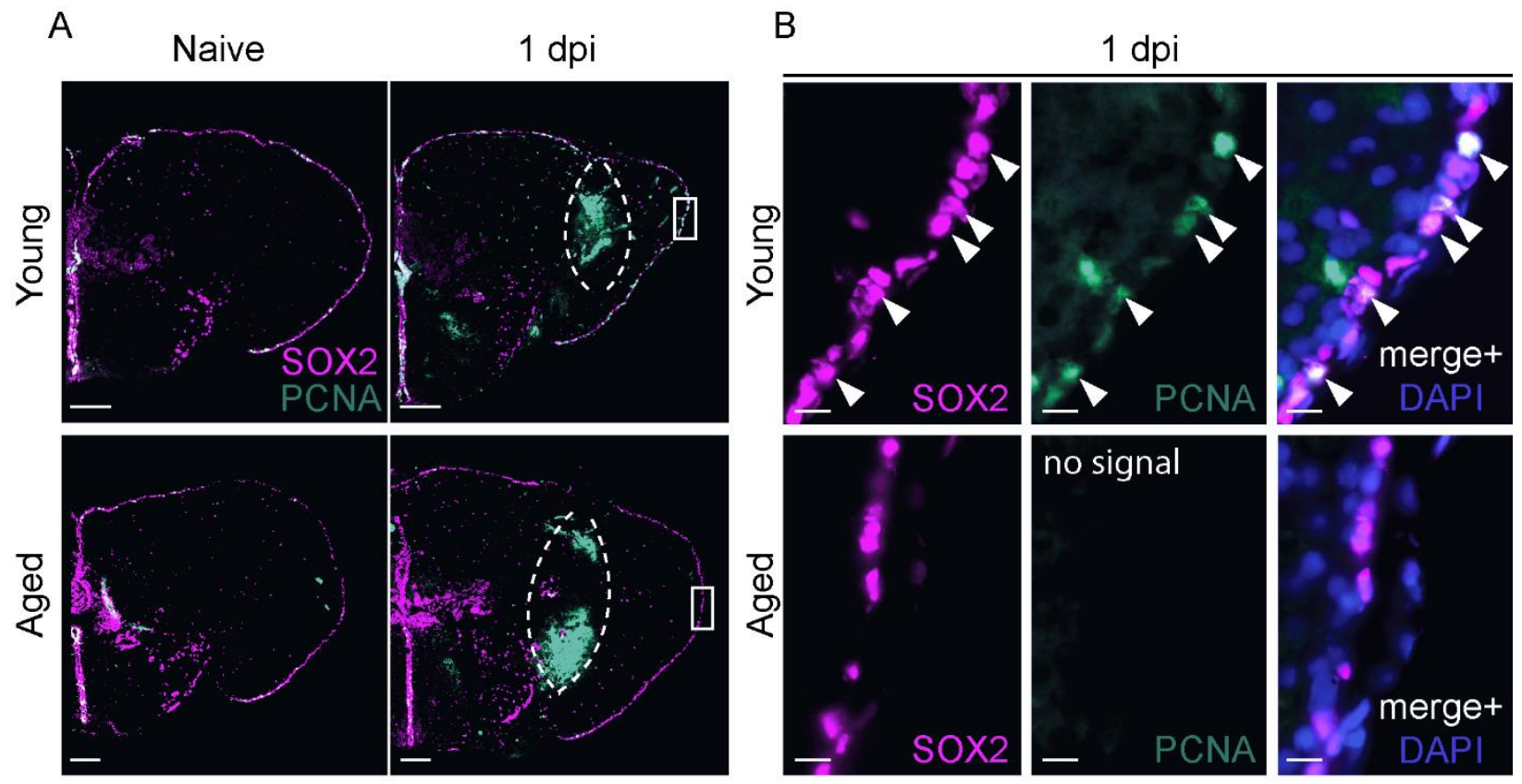

C

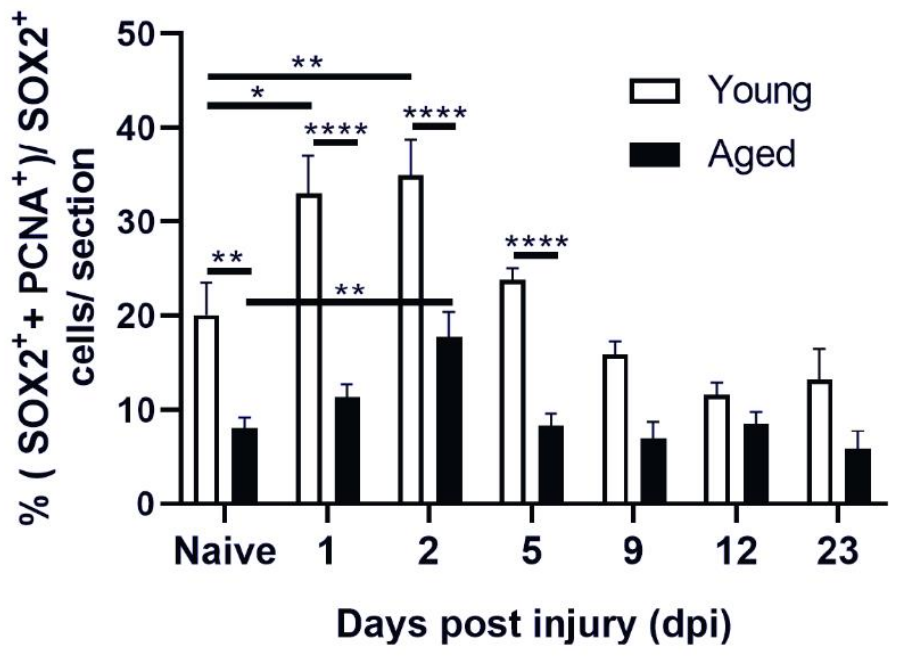

Figure 3 


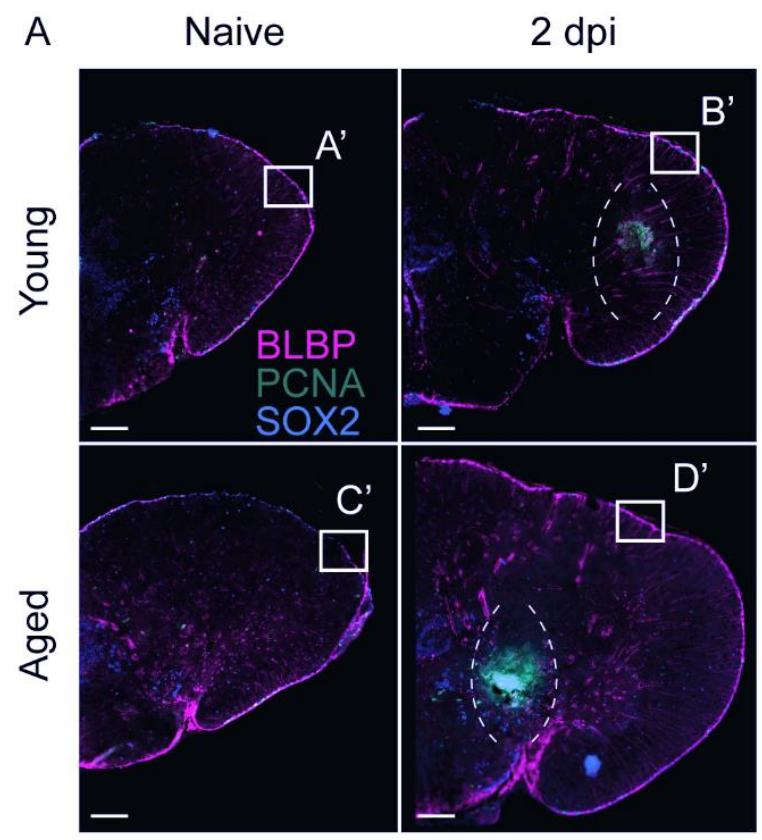

BLBP

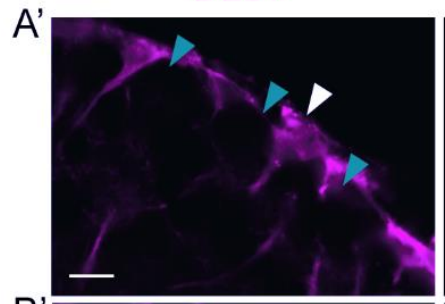

B'

C'

D'

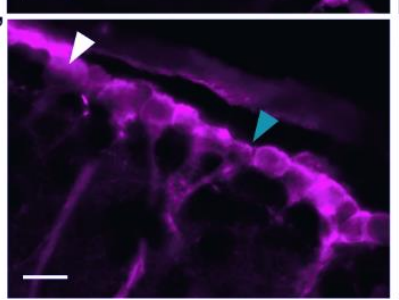

Figure 4
$\mathrm{B}$

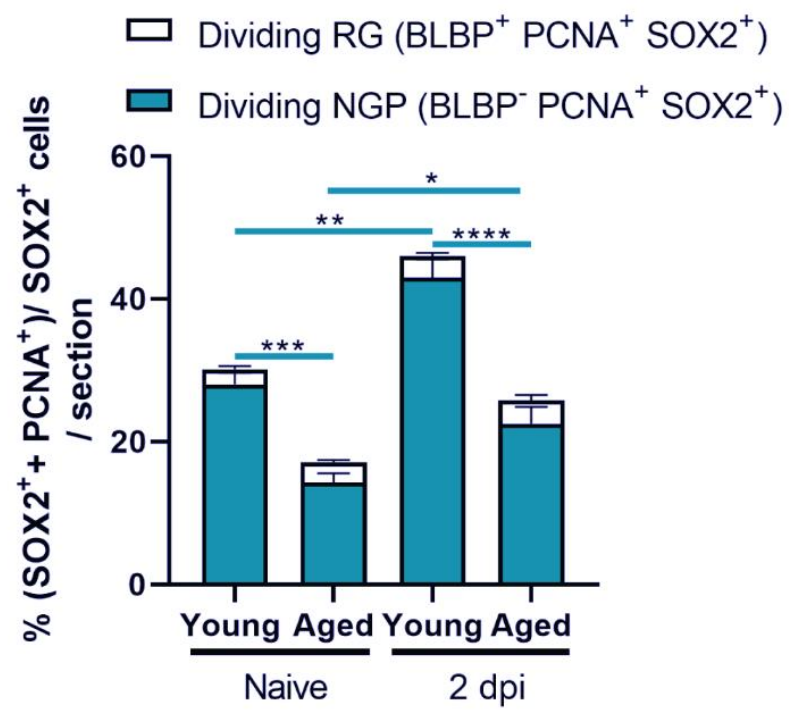

soX2

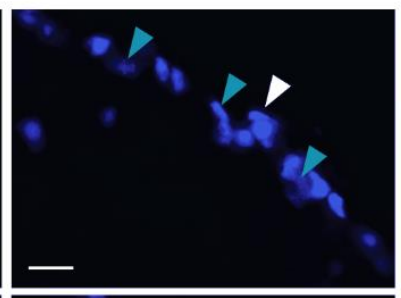

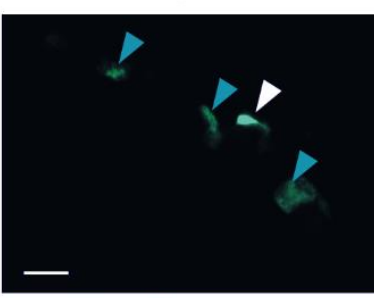
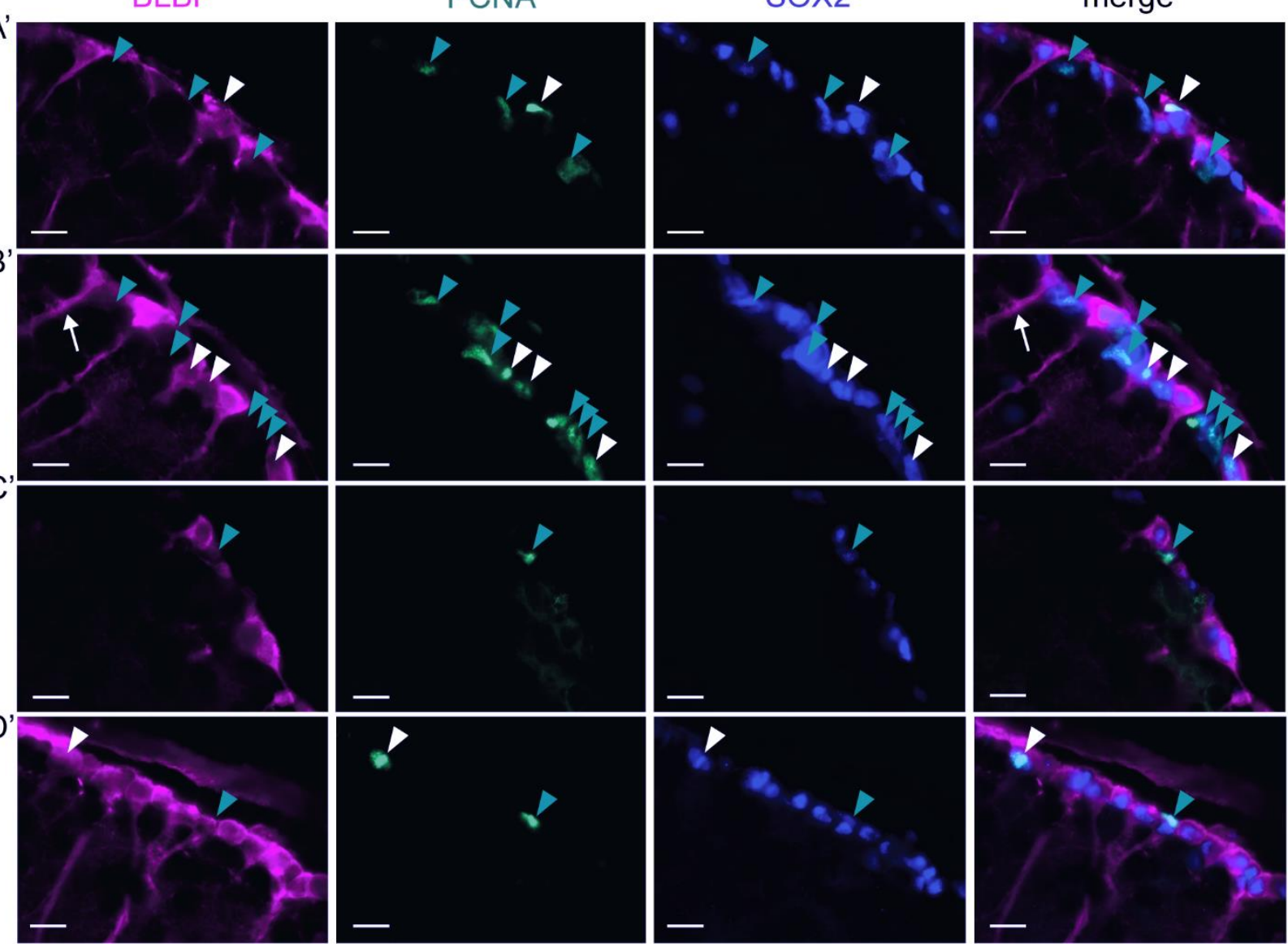


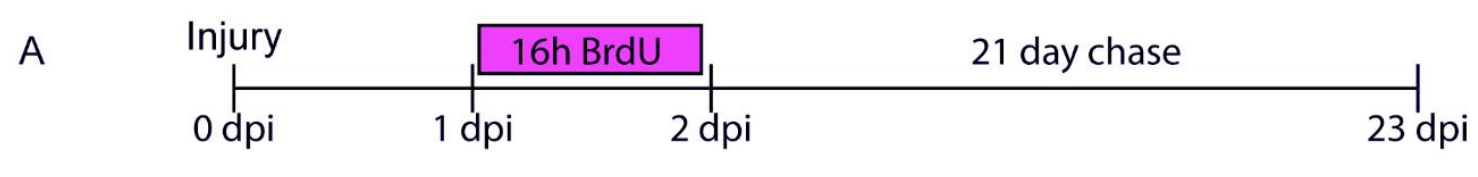

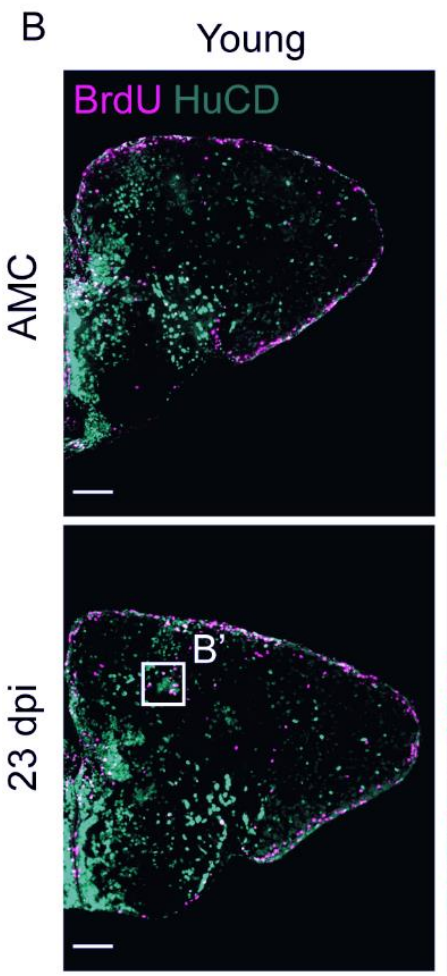

D

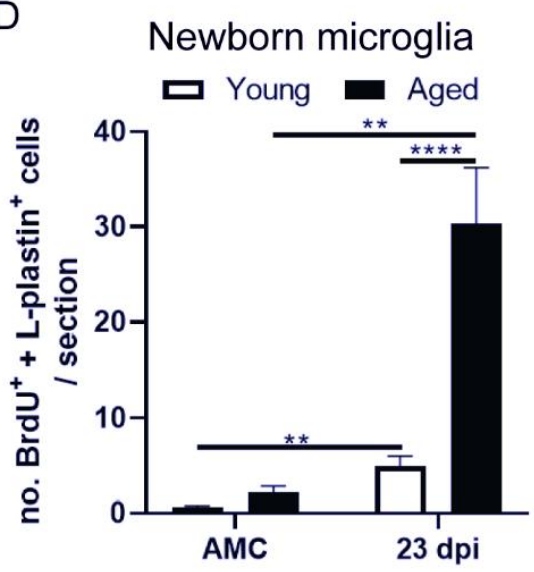

Aged

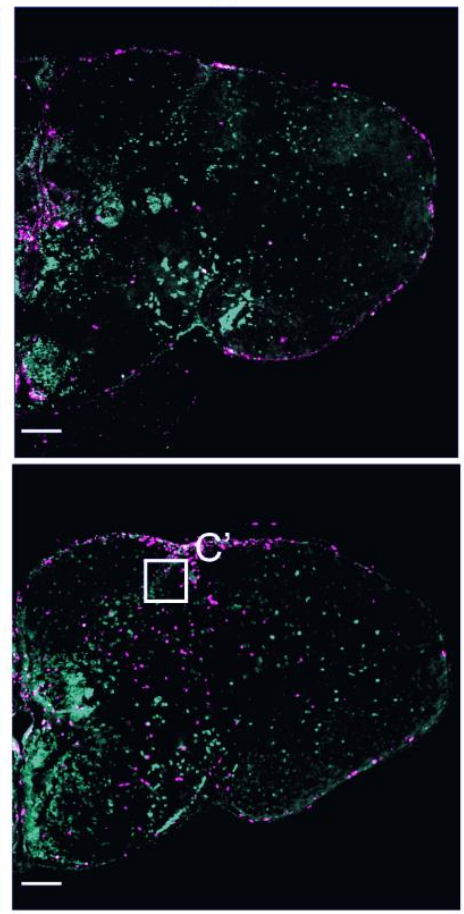

E

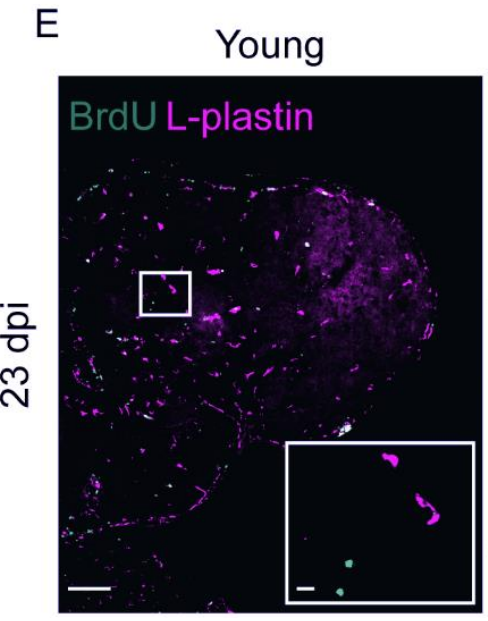

Newborn neurons

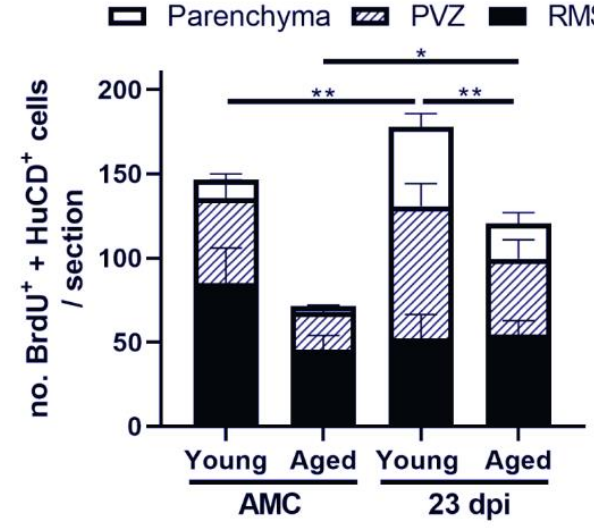

B' BrdU HuCD C' BrdU HuCD
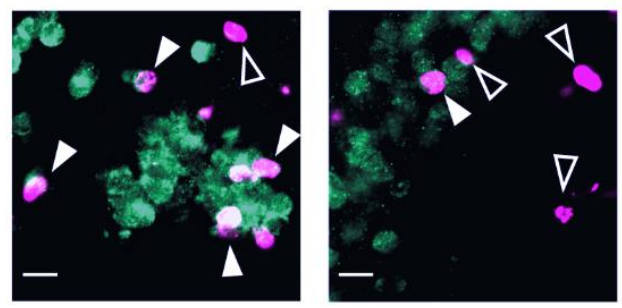

Aged

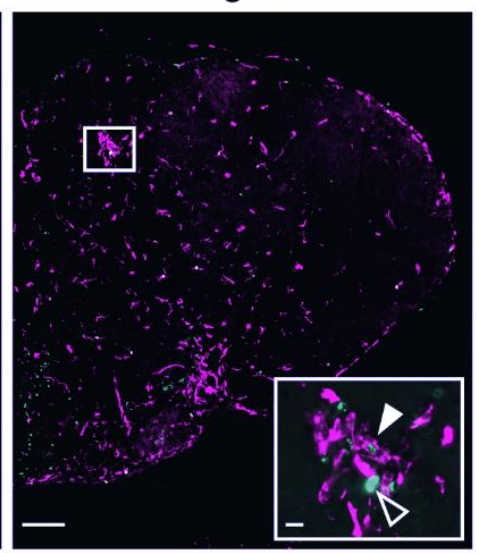

Figure 5 


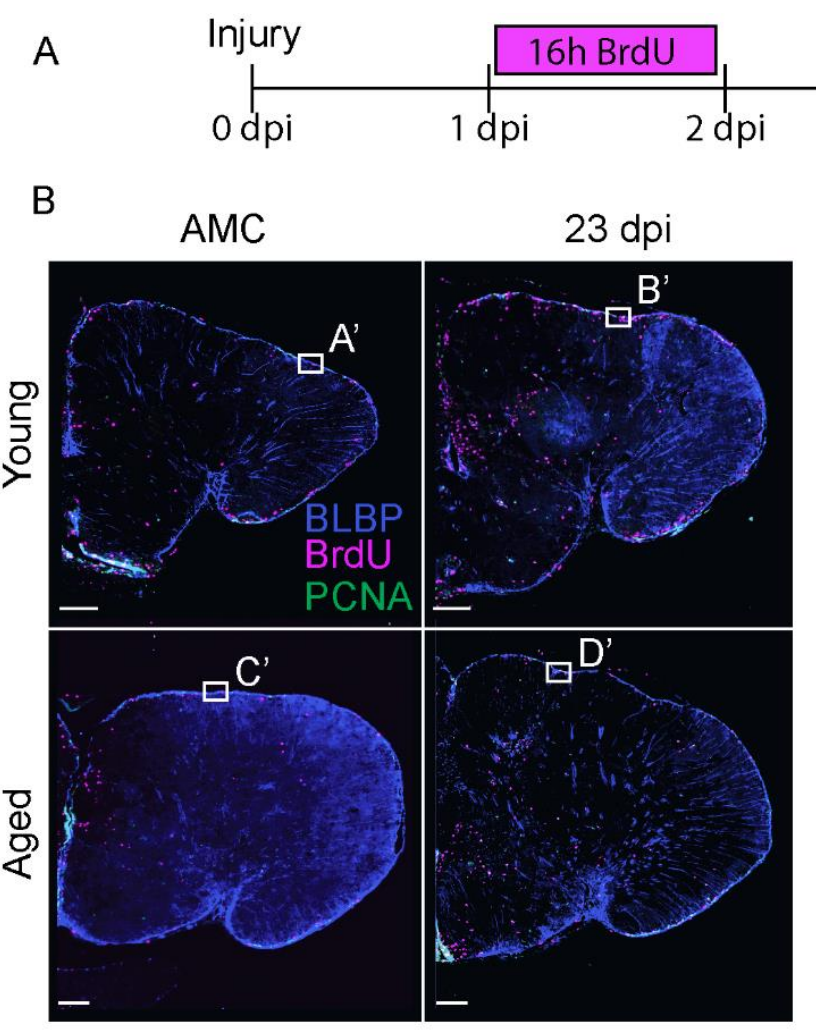

C
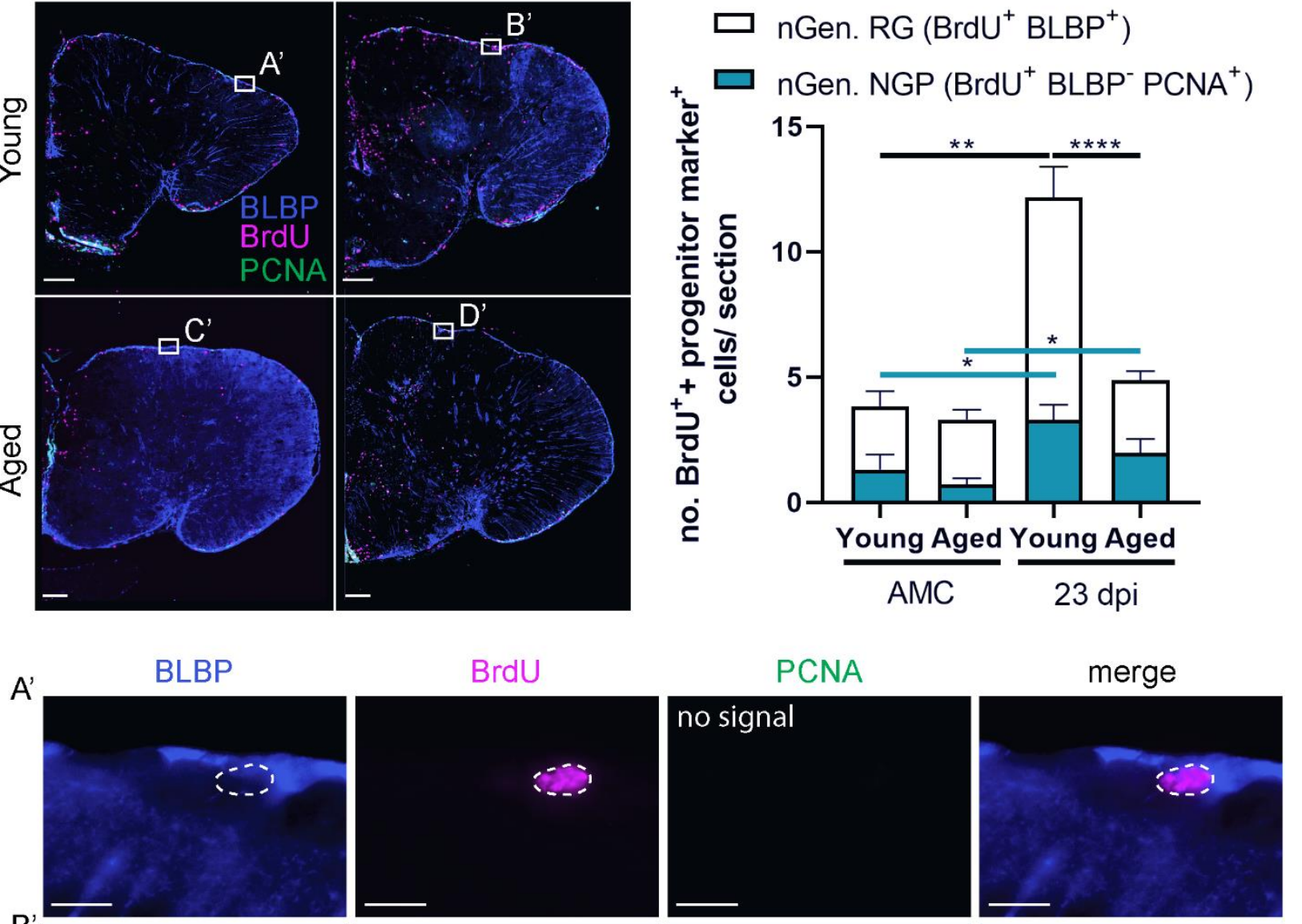

$B^{\prime}$
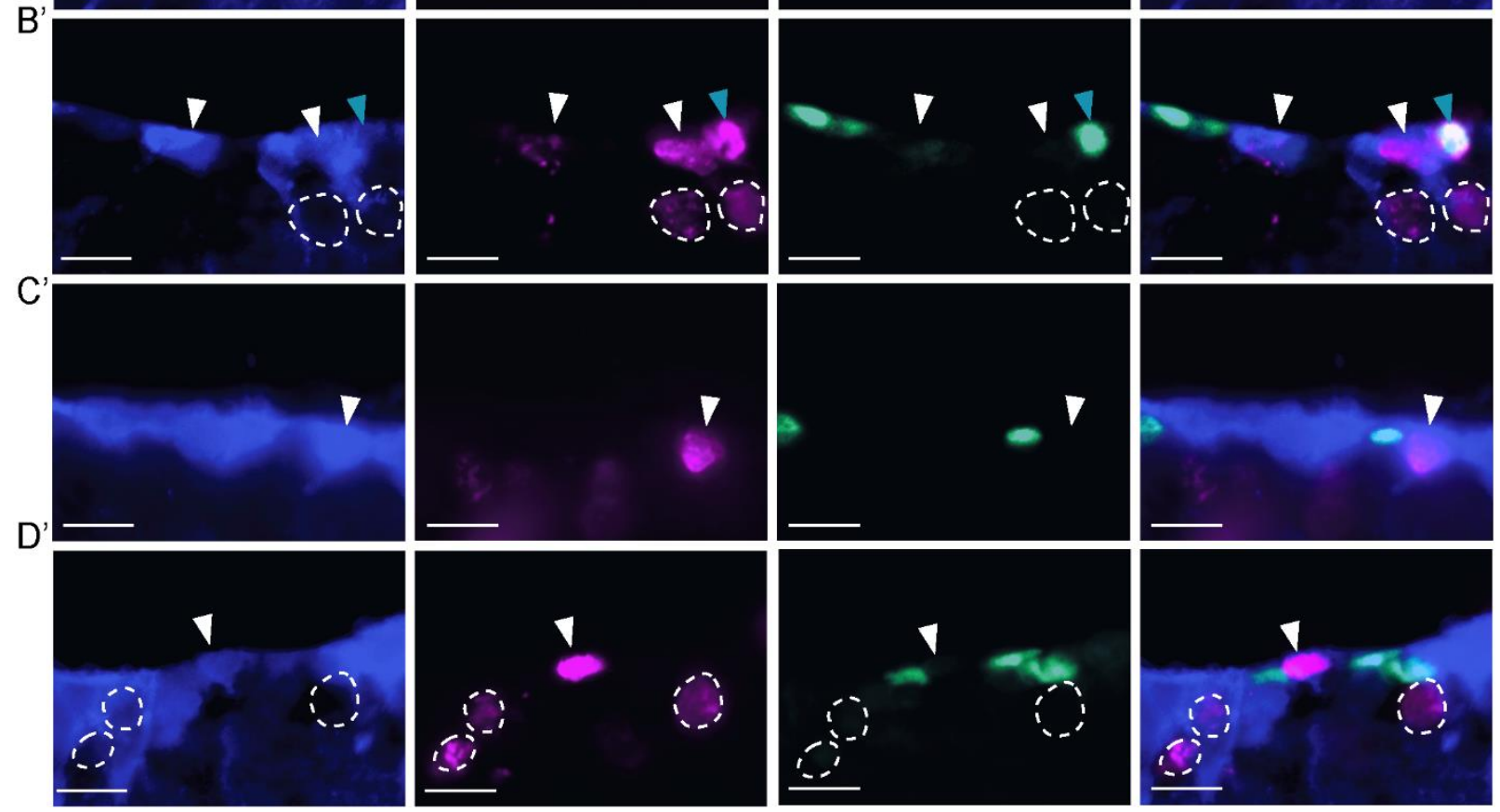


\section{$753 \quad$ Figure 6}

754 Figure legends

755 Figure 1: Aging impairs tissue recovery after brain injury

756 (A-D) Cresyl violet stainings of coronal sections illustrate the injury site and tissue recovery after 757 brain injury in the telencephalon of young killifish $(A, C)$ and of aged killifish $(B, D)$. At 30 dpi, the

758 aged telencephalon still shows a tissue scar in the parenchyma (arrowhead in $D^{\prime}$ ), while the 759 young telencephalon has no visible injury anymore. Of note, cells near the tissue scar appear 760 swollen ( $\left.D^{\prime}\right)$. Scale bar in A-D: $50 \mu \mathrm{m}$. Scale bar in $A^{\prime}-D^{\prime}: 20 \mu \mathrm{m}$.

761 (E) Quantification of the injury surface area (in $\mathrm{mm}^{2}$ ) measured at $1 \mathrm{hpi}$ and 1, 23, $30 \mathrm{dpi}$, in 762 young adult and aged killifish. At 1 hpi the injury size is similar between young adult and aged 763 killifish, towards 2 dpi the injury is significantly enlarged in aged fish. In addition, the injury is 764 still visible at 23 and $30 \mathrm{dpi}$, time points at which young adult fish demonstrate extensive 765 structural recovery.

766 (F) Countings of the absolute number of TUNEL ${ }^{+}$apoptotic cells detected in the injured telencephalon at 1, 2 and $5 \mathrm{dpi}$ reveal that apoptosis is significantly larger in aged fish at $1 \mathrm{dpi}$. *p $\leq 0,05$; Two-way ANOVA, followed by Sidak's multiple comparisons test. Values are mean \pm $S E M ; n \geq 4$. hpi: hours post injury, dpi: days post injury. Pictures of coronal brain sections in (A, $B, C, D)$ are also shown in Figure $S 2$ to illustrate how the injury surface area was measured, and in Figure S1 to visualize the DiD crystals and glial scarring at the site of injury.

772 Figure 2: The injury-induced inflammatory reaction is larger in the aged brain

773 (A) Staining for L-plastin (red) with DAPI (blue) on coronal brain sections of young adult and 774 aged killifish in naive conditions and at $2 \mathrm{dpi}$. Boxed areas are magnified in $\left(A^{\prime}-D^{\prime}\right)$. A more 775 pronounced increase in L-plastin ${ }^{+}$inflammatory cells is observed for aged than young adult

776 injured fish. $\left(A^{\prime}-D^{\prime}\right)$ Higher magnification of individual L-plastin ${ }^{+}$microglia/macrophages are 777 presented in the right bottom corner. Scale bars in $(A)$ and $\left(A^{\prime}-D^{\prime}\right): 100 \mu \mathrm{m}$. Scale bars of boxed 778 areas in $A^{\prime}-D^{\prime}: 10 \mu m$.

779 (B) Absolute number of L-plastin ${ }^{+}$microglia/macrophages in young adult and aged telencephali 780 in naive conditions and at $2 \mathrm{dpi}$. Both young adult and aged killifish show a significant increase 781 in inflammatory cells early after brain injury, but the number of microglia/macrophages is 782 significantly higher in aged fish at 1 and 2 dpi when compared to young adult individuals.

$783 * p \leq 0,05, * * p \leq 0,01, * * * p \leq 0,001, * * * * p \leq 0,0001$; One-way ANOVA is used to compare naive fish 784 to injured fish. Young: parametric one-way ANOVA, followed by Dunnett's multiple 785 comparisons test. Aged: non-parametric Kruskal-Wallis test, followed by Dunn's multiple 786 comparisons test. Two-way ANOVA is used to compare young and aged fish, followed by Sidak's 787 multiple comparisons test. Values are mean $\pm S E M ; n \geq 5$. dpi: days post injury. 
Figure 3: Aging diminishes neural progenitor cell proliferation in the ventricular zone of the killifish telencephalon, in naive conditions and in response to injury sections of young and aged killifish in naive conditions and at $1 \mathrm{dpi}$. The dashed lines encircle the site of injury filled with blood cells that autofluoresce in the green channel. (B) Magnifications of the boxed areas in A: Young adult killifish have a higher percentage of proliferating progenitor cells ( $\mathrm{SOX} 2^{+} \mathrm{PCNA}^{+}$cells, arrowheads) in the VZ compared to aged fish. Scale bars in A: $100 \mu \mathrm{m}$. Scale bars in $B: 10 \mu \mathrm{m}$. progenitor cells) in young adult and aged telencephali in naive conditions and at 1, 2, 5, 9, 12 and $23 \mathrm{dpi}$. At all time points investigated, young adult fish have a higher capacity for progenitor proliferation than aged fish. Interestingly, for both ages progenitor proliferation peaks at 2 dpi. $* p \leq 0,05, * * p \leq 0,01, * * * * p \leq 0,0001$; One-way ANOVA is used to compare naive fish to injured fish, followed by Dunnett's multiple comparisons test. Two-way ANOVA is used to compare young and aged fish at each time point, followed by Sidak's multiple comparisons test. Values are mean $\pm S E M ; n \geq 5$, except for aged, 9 dpi: $n=4$. VZ: ventricular zone, dpi: days post injury.

805 Figure 4: Aging reduces the proportion of dividing specialized NGPs, but not dividing common 806 RGs

807 (A) Triple staining for BLBP (magenta), PCNA (green) and SOX2 (blue) on coronal sections of young adult and aged killifish in naive conditions and at $2 \mathrm{dpi}$. The dashed lines encircle the site of injury filled with blood cells that autofluoresce in the green channel. Boxed areas are magnified in $\left(A^{\prime}-D^{\prime}\right)$. White arrowheads depict triple positive $B L B P^{+} S O X 2^{+} \mathrm{PCNA}^{+}$dividing $R G s$, while turquoise arrowheads mark double positive BLBP- SOX2 ${ }^{+}$PCNA $^{+}$dividing NGPs. $\left(C^{\prime}\right)$ Thicker RG fibers are noticed in young, injured fish (white arrow in $\mathrm{B}^{\prime}$ ), indicative for glial

813 activation. Young adult killifish clearly have more dividing NGPs compared to aged fish. In 814 addition, dividing RGs are only observed in small amounts in the VZ. Scale bars in (A): $100 \mu \mathrm{m}$. 815 Scale bars in $\left(A^{\prime}-D^{\prime}\right): 10 \mu \mathrm{m}$.

816 (B) Proportion of $\mathrm{BLBP}^{+} \mathrm{SOX2}{ }^{+} \mathrm{PCNA}^{+}$dividing RG and BLBP- SOX2 ${ }^{+} \mathrm{PCNA}^{+}$dividing NGPs over all 817 SOX $2^{+}$cells (all progenitor cells) in the VZ of young and aged telencephali in naive conditions 818 and at 2 dpi. Reactive progenitor proliferation is mainly supported by specialized NGPs and is 819 highest in young adult killifish. Aged killifish have significantly lower percentages of dividing 820 NGPs in both naive and injured conditions. ${ }^{*} p \leq 0,05,{ }^{*} p \leq 0,01, * * * p \leq 0,001, * * * * p \leq 0,0001$;

821 Unpaired t-test is used to compare naive fish to injured fish. Two-way ANOVA is used to 822 compare young and aged fish, followed by Sidak's multiple comparisons test. Values are mean \pm 823 SEM; $n \geq 5$. RG: radial glia, NGP: non-glial progenitor, VZ: ventricular zone, dpi: days post injury. 
824 Figure 5: Aged killifish generate less newborn neurons but more newborn microglia/macrophages in the parenchyma of the telencephalon compared to young ault killifish

827 (A) Experimental set up: Day 0: time of a stab-wound injury; From 1 to 2 dpi: injured fish and AMCs are placed in BrdU water for 16 hours: BrdU will be incorporated in the DNA of dividing cells and passed on to the progeny upon each cell division. $23 \mathrm{dpi}$ : regeneration is completed in young fish, all brain samples of young and aged, AMC and injury conditions are collected for cell analysis (as illustrated in B-E).

832 (B) Double staining for BrdU (magenta) and HuCD (green). Boxed areas are magnified in ( $\left.B^{\prime}, C^{\prime}\right)$. White closed arrowheads depict double positive $\mathrm{BrdU}^{+} \mathrm{HuCD}^{+}$newborn neurons; open arrowheads indicate newborn single positive $\mathrm{BrdU}^{+}$cells of unknown cell type. While the BrdU signal overlaps with HuCD (neuronal marker) in young adult killifish, representing newborn neurons, aged killifish mostly have BrdU positive cells lying next to $\mathrm{HuCD}^{+}$neurons, suggesting these cells are another cell type. Scale bars in (B): $100 \mu \mathrm{m}$. Scale bars in $\left(B^{\prime}, C^{\prime}\right): 10 \mu \mathrm{m}$. (C) Number of $\mathrm{BrdU}^{+} \mathrm{HuCD}^{+}$newborn neurons near the RMS of the subpallium, in the PVZ of the dorsal pallium, and in the parenchyma. In the parenchyma, significantly lower numbers of newborn neurons are present in aged injured fish compared to young adult injured fish. (D) Number of BrdU+ L-plastin ${ }^{+}$newborn microglia/macrophages in the parenchyma. Aged injured fish generate large numbers of newborn microglia/macrophages by $23 \mathrm{dpi}$. $p \leq 0,05$, $* * p \leq 0,01, * * * * p \leq 0,0001$; Unpaired t-test or non-parametric Mann Whitney test is used to compare AMC to $23 \mathrm{dpi}$ fish. Two-way ANOVA is used to compare young and aged fish, followed by Sidak's multiple comparisons test. Values are mean $\pm S E M ; n \geq 5$. right corner of each panel. A cluster of L-plastin+ microglia/macrophages is visible in the parenchyma of aged injured fish, but not in young injured fish, at $23 \mathrm{dpi}$. White closed arrowhead depicts a $\mathrm{BrdU}^{+} \mathrm{L}_{\text {-plastin }}{ }^{+}$newborn microglia/macrophages, inside this cluster. The open arrowhead points to a green autofluorescent blood cell recognizable by its oval shape and visible nucleus. Scale bars in (E): $100 \mu \mathrm{m}$. Scale bars of boxed areas: $10 \mu \mathrm{m}$. 16h: 16 hours, AMC: age-matched control, RMS: rostral migratory stream, PVZ: periventricular zone, dpi: days post

853 injury.

\section{Figure 6: Aging hampers the replenishing of progenitors in the VZ after injury}

855 (A) Experimental set up: Day 0: time of a stab-wound injury; From 1 to 2 dpi: injured fish and AMCs are placed in BrdU water for 16 hours: BrdU will be incorporated in the DNA of dividing cells and passed on to the progeny upon each cell division. $23 \mathrm{dpi}$ : regeneration is completed in

858 young fish, all brain samples of young and aged, AMC and injury conditions are collected for cell 859 analysis (as illustrated in B).

860 (B) Triple staining for BLBP (blue), BrdU (magenta) and PCNA (green). Boxed areas are 861 magnified in $\left(A^{\prime}-D^{\prime}\right)$. White arrowheads depict double positive $\mathrm{BLBP}^{+} \mathrm{BrdU}^{+}$newly generated 
862 RGs. Turquoise arrowheads mark double positive BLBP- BrdU $^{+}$PCNA $^{+}$newly generated NGPs.

863 Newly generated RGs, and to a lesser extent newly generated NGPs, are produced after injury 864 in the VZ. Scale bars in (B): $100 \mu \mathrm{m}$. Scale bars in $\left(A^{\prime}-D^{\prime}\right): 10 \mu \mathrm{m}$.

865 (C) Number of $\mathrm{BLBP}^{+} \mathrm{BrdU}^{+}$newly generated RGs and $\mathrm{BLBP}^{-} \mathrm{BrdU}^{+} \mathrm{PCNA}^{+}$newly generated NGPs 866 in the VZ of young and aged telencephali in naive conditions (AMC) and at $23 \mathrm{dpi}$. Significantly 867 more newly generated progenitors are produced after injury. Young adult fish create 868 significantly more newly generated RGs compared to aged killifish at $23 \mathrm{dpi}$. This difference is 869 not significant for newly generated NGPs. ${ }^{*} p \leq 0,05, * * p \leq 0,01, * * * * p \leq 0,0001$; Unpaired t-test or 870 non-parametric Mann Whitney test is used to compare AMC to $23 \mathrm{dpi}$ fish. Two-way ANOVA is 871 used to compare young and aged fish, followed by Sidak's multiple comparisons test. Values are 872 mean \pm SEM; $n \geq 5$. RG: radial glia, NGP: non-glial progenitor, AMC: age-matched control, VZ: 873 ventricular zone, dpi: days post injury, nGen: newly generated.

\section{Supplementary figure legends}

\section{Supplementary figure 1: Research methodology and glial scarring}

876 (A) Survival curve of $N$. furzeri females (strain GRZ-AD), housed in a Tecniplast ZebTEC multi877 linking aquarium system $(n=48) .6$ week-old killifish are chosen as young adult based on $100 \%$ 878 survival rate and having reached sexual maturity. 18 week-old killifish are chosen as aged based 879 on $76,6 \%$ survival rate and showing phenotypic aging hallmarks (illustrated in B).

880 (B) Photographs of a young adult (6 week-old) and aged (18 week-old) N. furzeri females (strain GRZ-AD). Aged females show phenotypic aging hallmarks, such as a spinal curvature and

882 protrusion of the lip (arrowheads).

883 (C) Schematic of the stab-wound injury method. After removing skin above the skull, a 33-gauge 884 Hamiliton needle is pushed into the medial zone of the right telencephalic hemisphere. The 885 needle is dipped in DiD solution (red), which labels the membranes of cells around the injury 886 site (illustrated in D,E).

887 (D-K) The injury site can be found by scanning sections for DiD-positivity (red crystals) after 888 cryosectioning, for example at $30 \mathrm{dpi}$. (D,F,H, J) Adjacent coronal sections of a young adult 889 killifish telencephalon. (D) The DiD dye is still visible at $30 \mathrm{dpi}$. (F) Cresyl violet staining shows 890 that the tissue of the young adult fish is structurally regenerated. (H) No glial scar (or 891 malformation) is visible after staining for L-plastin ${ }^{+}$microglia/macrophages in the young adult 892 brain at the site of injury. ( $\mathrm{J}$ ) Gs${ }^{+} \mathrm{RG}$ fiber distribution is normal in young adult fish at $30 \mathrm{dpi}$. 893 (E,G,I) Adjacent coronal sections of an aged killifish telencephalon. (E) The DiD dye clearly 894 marks the injury site at 30 dpi. (G) Cresyl violet staining shows tissue scarring (arrowhead in $\mathrm{G}^{\prime}$ ), 895 indicative for incomplete repair. (I) Signs of glial scarring are visible after staining for L-plastin ${ }^{+}$ 896 microglia/macrophages in the aged brain at the site of injury. (K) Gs ${ }^{+}$RG fibers are in close 897 contact with the glial scar (arrowhead in K'). 
$898\left(D^{\prime}, E^{\prime}, F^{\prime}, G^{\prime}, H^{\prime}, I^{\prime}\right)$ are magnifications of the boxed area in $(D, E, F, G, H, I)$ respectively. Scale bars in 899 D-I: $100 \mu \mathrm{m}$. Scale bars in $D^{\prime}-I^{\prime}: 50 \mu \mathrm{m}$. dpi: days post injury.

900 Supplementary figure 2: Methodology of injury surface area measurements

901 In Image (FIJI) the scale is set and a polygon is drawn around the injury site with the polygon 902 tool (blue line). Image calculates the surface of the polygon. At early stages, the injury site is

903 visible by a blood clot and the borders of this clot are taken as the injury borders (young, aged 904 at $1 \mathrm{dpi}$, scale bars: $50 \mu \mathrm{m}$ ). In later stages, only a malformation of the parenchymal tissue is 905 visible (aged $30 \mathrm{dpi}$, scale bar: $50 \mu \mathrm{m}$ ). In this case, the borders of this malformation are taken 906 as the injury borders (Illustrated in the inset of aged $30 \mathrm{dpi}$, scale bar: $20 \mu \mathrm{m}$ ).

907 Supplementary figure 3: Injury induces proliferation of microglia in young and aged fish

908 (A) Double staining for L-plastin (magenta) and PCNA (green) with DAPI (blue) shows 909 proliferating microglia/macrophages (arrowhead) on coronal sections of young and aged 910 killifish at 2 dpi. Scale bars: $10 \mu \mathrm{m}$.

911 (B) Absolute number of double positive L-plastin ${ }^{+}$PCNA ${ }^{+}$proliferating microglia/macrophages in 912 young adult and aged telencephali in naive conditions and at 1 and 2 dpi.

913 Microglia/macrophages start proliferating early after injury in the killifish telencephalon.

914 Significantly higher levels of proliferating microglia/macrophages are observed at 1 and 2 dpi

915 for both ages. This activation is slightly more pronounced but highly variable in aged killifish 916 compared to young adult fish. ${ }^{*} p \leq 0,05,{ }^{*} p \leq 0,01$; One-way ANOVA is used to compare naive 917 fish to injured fish for each. Young: parametric one-way ANOVA, followed by Dunnett's multiple 918 comparisons test. Aged: non-parametric Kruskal-Wallis test, followed by Dunn's multiple 919 comparisons test. Two-way ANOVA is used to compare young and aged fish, followed by Sidak's 920 multiple comparisons test. Values are mean $\pm S E M ; n \geq 5$.

\section{Supplementary figure 4: Counting method}

922 Schematic view of the different regions in which different types of cells are counted in the right 923 telencephalic hemisphere. VZ: ventricular zone, PVZ: periventricular zone, RMS: rostral

924 migratory stream, RG: radial glia, NGP: non-glial progenitor. Created with BioRender.com.

925 Supplementary table 1: Percentages of dividing RGs and NGPs among the total amount of 926 dividing progenitors differ between young adult and aged killifish

927 Independent of injury, aged killifish display more dividing RGs in regard to the total amount of

928 dividing progenitors compared to young adult fish. * $p \leq 0,05$; Unpaired T-test is used to compare 929 the percentage of dividing RGs or NGPs among all dividing progenitors between young adult 930 and aged killifish. Values are mean \pm SEM; $n \geq 5$.

931 Supplementary figure 5: The dividing progenitor pool is not depleted within a short period 932 after injury

933 (A) Absolute number of $\mathrm{BLBP}^{+} \mathrm{PCNA}^{+}$dividing RGs and (B) BLBP- PCNA ${ }^{+}$dividing NGPs in the VZ 
934 of young and aged telencephali in naive conditions (AMC) and at $23 \mathrm{dpi}$. Even after the

935 production of neurons, the number of dividing RGs and NGPs is respectively similar or increased

936 at $23 \mathrm{dpi}$ compared to AMCs. This suggests that killifish replenish the proliferative stem cell

937 pool after injury. ${ }^{*} p \leq 0,05, * * p \leq 0,01$, Unpaired t-test or non-parametric Mann Whitney test is

938 used to compare AMC to $23 \mathrm{dpi}$ fish. Two-way ANOVA is used to compare young and aged fish,

939 followed by Sidak's multiple comparisons test. Values are mean $\pm S E M ; n \geq 5$. RG: radial glia,

940 NGP: non-glial progenitor, AMC: age-matched control, VZ: ventricular zone, dpi: days post

941 injury. 